\title{
GALOIS AND BIGALOIS OBJECTS OVER MONOMIAL NON SEMISIMPLE HOPF ALGEBRAS
}

\author{
JULIEN BiCHON \\ Laboratoire de Mathématiques Appliquées, Université de Pau et des Pays de l'Adour, \\ IPRA, Avenue de l'université, 64000 Pau, France. E-mail: Julien.Bichon@univ-pau.fr
}

\begin{abstract}
We describe the Hopf-Galois extensions of the base field and the biGalois groups of non semisimple monomial Hopf algebras. The main feature of our description is the use of modified versions of the second cohomology group of the grouplike elements. Our computations generalize the previous ones of Masuoka and Schauenburg for the Taft algebras.
\end{abstract}

\section{Introduction}

This paper contributes to the general problem of determining the Hopf-Galois extensions of a given Hopf algebra. We describe here the Hopf-Galois extensions (of the base field) and the biGalois groups of an important class of pointed Hopf algebras, namely the monomial non semisimple Hopf algebras, which were recently classified by Chen, Huang, Ye and Zhang [6].

The first classification results of Hopf-Galois extensions for a non commutative and non cocommutative Hopf algebra are due to Masuoka [12, without any restriction on the subring of invariants, in the case of the Taft algebras. Then Schauenburg 16] determined all Hopf-Galois extensions of the base field (= Galois objects) for the general Taft algebras with several grouplike elements, and computed their biGalois groups. The generalized Taft algebras are monomial Hopf algebras, so our results generalize Schauenburg's ones. The main feature of our description of Galois objects and biGalois groups for monomial Hopf algebras is the use of modified versions of the second cohomology group of the grouplike elements. It gives a general concise descriptive formula for the set of isomorphism classes of Galois objects and for the biGalois groups, leaving the explicit computations to be done at the cohomology group level.

Our computations do not use the underlying quiver of a monomial Hopf algebra: it certainly would be interesting to get a connection between our description and

2000 Mathematics Subject Classification: 16W30, 20 J06. 
the properties of the quiver. Also it would interesting to describe Galois objects and biGalois groups over the Hopf algebra associated to a Hopf quiver by Cibils and Rosso in [7].

This paper is organized as follows. The main definitions and results around HopfGalois extensions and biGalois groups are recalled in the first section. We also recall the notion of a group datum of [6], which by the results of [6] is equivalent to the notion of a monomial Hopf algebra, and divide the various group data into six different types. Finally this preliminary section is concluded with some cohomogical preliminaries: the definition of the modified second cohomology group, to be used in the description results of the next sections. In Section 2, we describe the Galois objects over the (monomial) Hopf algebra associated with a group datum, the type VI case being treated thanks to the results of the third section. The very end of the section deals with the classification up to homotopy of these Galois extensions, a concept recently introduced by Kassel and Schneider [10]: this is an easy task using the results of [10]. Section 3 contains the general description of the BiGalois group of a monomial Hopf algebra. In Section 4, some explicit examples are examined in detail: Hopf algebras associated with cyclic group data, including the Taft algebras and the simple-pointed Hopf algebras, and Hopf algebras associated with decomposable group data, including the generalized Taft algebras with several grouplike. In particular the results of Masuoka [12] and Schauenburg [16] are recovered.

Throughout this paper $k$ is a commutative field containing all primitive roots of unity. This forces $k$ to be a characteristic zero field. The multiplicative group of non-zero elements of $k$ is denoted by $\dot{k}$. For $n \in \mathbb{N}^{*}$, the group of nth-roots of unity in $k$ is denoted by $\mu_{n}$. By our assumption we have $\left|\mu_{n}\right|=n$.

\section{Preliminaries}

\section{$1.1 \quad$ Hopf-Galois extensions}

We first recall the principal facts concerning Hopf-Galois extensions, the main object of study in this paper. The book [13] is a convenient reference for this topic.

Let $A$ be a Hopf algebra. A right $A$-Galois extension (of $k$ ) is a non-zero right $A$-comodule algebra $Z$ such that the linear map $\kappa_{r}$ defined by the composition

$$
\kappa_{r}: Z \otimes Z \stackrel{1_{Z} \otimes \alpha}{\longrightarrow} Z \otimes Z \otimes A \stackrel{m_{Z} \otimes 1_{A}}{\longrightarrow} Z \otimes A
$$

where $\alpha$ is the coaction of $A$ and $m_{Z}$ is the multiplication of $Z$, is bijective. We also say that a right $A$-Galois extension (of $k$ ) is an $A$-Galois object. A morphism of $A$ Galois objects is an $A$-colinear algebra morphism. It is known that any morphism of $A$-Galois extensions is an isomorphism. The set of isomorphism classes of $A$-Galois objects is denoted by $\operatorname{Gal}(A)$. For example, when $A=k[G]$ is a group algebra, we have $\operatorname{Gal}(k[G]) \cong H^{2}(G, \dot{k})$. However $\operatorname{Gal}(A)$ does not carry a natural group structure in general. 
Amongst $A$-Galois extensions, the cleft ones will be especially important in this paper. First recall that a 2-cocycle over $A$ is a convolution invertible linear map $\sigma: A \otimes A \longrightarrow k$ satisfying

$$
\sigma\left(a_{(1)}, b_{(1)}\right) \sigma\left(a_{(2)} b_{(2)}, c\right)=\sigma\left(b_{(1)}, c_{(1)}\right) \sigma\left(a, b_{(2)} c_{(2)}\right)
$$

and $\sigma(a, 1)=\sigma(1, a)=\varepsilon(a)$, for all $a, b, c \in A$. For example, when $A=k[G]$ is a group algebra, a cocycle over $A$ corresponds precisely to an element of $Z^{2}(G, \dot{k})$. To any 2-cocycle $\sigma$ on $A$ we associate the right $A$-comodule algebra ${ }_{\sigma} A$. As an $A$-comodule ${ }_{\sigma} A=A$ and the product of $\sigma A$ is defined to be

$$
a_{\sigma} \cdot b=\sigma\left(a_{(1)}, b_{(1)}\right) a_{(2)} b_{(2)}, \quad a, b \in A .
$$

Let $Z$ be a right $A$-comodule algebra. Then $Z$ is said to be cleft if one the following equivalent conditions holds:

- There exists a 2 -cocycle over $A$ such that $Z \cong{ }_{\sigma} A$ as $A$-comodule algebras.

- $Z$ is $A$-Galois and $Z \cong A$ as $A$-comodules.

- There exists an $A$-colinear convolution invertible map $A \longrightarrow Z$.

The equivalence of the above assertions are due to Doi and Takeuchi and to Blattner and Montgomery. We refer the reader to [13. for proofs and for the original references. It is known that any $A$-Galois extension of $k$ is cleft if $A$ has one of the following properties:

- $A$ is finite-dimensional (by [11]),

- $A$ is pointed, i.e. all its simple comodules are one-dimensional, (by [9], Remark $10)$.

Since we only consider pointed (and finite-dimensional) Hopf algebras in this paper, we only consider cleft Galois objects. The reader might consult [3] for examples of non cleft Galois objects.

Although this will not be needed in this paper, the reader might like to know about the categorical interpretation of Hopf-Galois extensions, which is especially enlightening when we have a view towards biGalois objects. Let $\operatorname{Comod}(A)$ be the category of left $A$-comodules. Recall [18, that a fibre functor $\omega: \operatorname{Comod}(A) \longrightarrow$ $\operatorname{Vect}(k)$ is a monoidal $k$-linear exact and faithful functor commuting with colimits. Let $Z$ be a right $A$-Galois extension of $k$ : Ulbrich [18 associates a fibre functor $\omega_{Z}$ to such a Galois extension. This fibre functor is defined by $\omega_{Z}(V)=Z \square_{A} V$ where $Z \square_{A} V$ is the cotensor product over $A$ of the right $A$-comodule $Z$ and of the left $A$-comodule $V$. Then Ulbrich shows in [18 that this defines a category equivalence between $A$-Galois extensions of $k$ and fibre functors over $\operatorname{Comod}(A)$. The interpretation of the notion of cleft extension is particularly nice in this setting. Let $\omega$ be a fibre functor on $\operatorname{Comod}(A)$. Then the following assertions are equivalent: - The Galois extension corresponding to the fibre functor $\omega$ is cleft.

- The fibre functor $\omega$ is isomorphic, as a functor, with the forgetful functor.

- The fibre functor $\omega$, when restricted to finite-dimensional $A$-comodules, preserves the dimensions of the underlying vector spaces. 
The hard part when showing the equivalence of the above assertions is to see that the third one implies the second one. This a consequence of a result of Etingof and Gelaki (Proposition 4.2.2 in [5]) combined with Tannaka-Krein type results.

Let us now come to biGalois extensions. Similarly to the right case, a left $A$ Galois extension (of $k$ ) is a non-zero left $A$-comodule algebra $Z$ such that the linear map $\kappa_{l}$ defined by the composition

$$
\kappa_{l}: Z \otimes Z \stackrel{\beta \otimes 1_{Z}}{\longrightarrow} A \otimes Z \otimes Z \stackrel{1_{A} \otimes m_{Z}}{\longrightarrow} A \otimes Z
$$

where $\beta$ is the coaction of $A$ and $m_{Z}$ is the multiplication of $Z$, is bijective.

Let $A$ and $B$ be Hopf algebras. An algebra $Z$ is said to be an $A$-B-bigalois extension [15] if $Z$ is both a left $A$-Galois extension and a right $B$-Galois extension, and if $Z$ is an $A$-B-bicomodule.

Now consider a Hopf algebra $A$ and an $A$-Galois object $Z$. Then Schauenburg shows (Theorem 3.5 in [15]) that there exists a Hopf algebra $L(Z, A)$ such that $Z$ is an $L(Z, A)$-A-biGalois extension. The Hopf algebra $L(Z, A)$ is unique: if $B$ is another Hopf algebra such that $Z$ is $B$-A-biGalois, there exists a unique Hopf algebra isomorphism $f: L(Z, A) \rightarrow B$ such that $\left(f \otimes \operatorname{id}_{Z}\right) \circ \beta=\beta^{\prime}$, where $\beta$ and $\beta^{\prime}$ denote the respective left coactions of $L(Z, A)$ and $B$. In fact the Hopf algebra $L(Z, A)$ is the Tannaka-Krein reconstructed object from the fibre functor associated with the $A$-Galois extension $Z$ : this is Theorem 5.5 in [15]. In particular the $k$-linear monoidal comodule categories over two Hopf algebras $A$ and $B$ are equivalent if and only if there exists an $A$ - $B$-biGalois extension. More precisely by Corollary 5.7 of [15] there is a category equivalence between:

- The category of $k$-linear monoidal equivalences of categories between $\operatorname{Comod}(A)$ and $\operatorname{Comod}(B)$.

- The category of $A-B$ biGalois extensions.

Thus $\operatorname{BiGal}(A)$, the biGalois group of $A$, defined by Schauenburg in 15 as the set of isomorphism classes of $A$-biGalois extensions (i.e. $A$ - $A$-biGalois extensions) endowed with the cotensor product, might also be seen as the group of monoidal isomorphism classes of monoidal $k$-linear auto-equivalences of the cate$\operatorname{gory} \operatorname{Comod}(A)$. In this way if there exists an $A$ - $B$-biGalois extension, then there is a bijection $\operatorname{Gal}(A) \cong \operatorname{Gal}(B)$ and a group isomorphism $\operatorname{BiGal}(A) \cong \operatorname{BiGal}(B)$. For example, when $A=k[G]$ is a group algebra, we have a group isomorphism $\operatorname{BiGal}(k[G]) \cong \operatorname{Aut}(G) \ltimes H^{2}(G, \dot{k})$.

\subsection{Group data and monomial Hopf algebras}

Following Chen, Huang, Ye and Zhang [6], we define a group datum (over $k$ ) to be a quadruplet $\mathbb{G}=(G, g, \chi, \mu)$ consisting of a finite group $G$, a central element $g \in G$, a character $\chi: G \longrightarrow \dot{k}$ with $\chi(g) \neq 1$ and an element $\mu \in k$ such that $\mu=0$ if $o(g)=o(\chi(g))$, and that if $\mu \neq 0$, then $\chi^{o(\chi(g))}=1$.

We need to classify the various group data into six different types. 
- A type I group datum $\mathbb{G}=(G, g, \chi, \mu)$ is a group datum with $\mu=0, d=$ $o(\chi(g))=o(g)$ and $\chi^{d}=1$. In this case we simply write $\mathbb{G}=(G, g, \chi)$.

- A type II group datum $\mathbb{G}=(G, g, \chi, \mu)$ is a group datum with $\mu=0, d=$ $o(\chi(g))=o(g)$ and $\chi^{d} \neq 1$. In this case we simply write $\mathbb{G}=(G, g, \chi)$.

- A type III group datum $\mathbb{G}=(G, g, \chi, \mu)$ is a group datum with $\mu=0, d=$ $o(\chi(g))<o(g)$ and $\chi^{d}=1$. In this case we simply write $\mathbb{G}=(G, g, \chi)$.

- A type IV group datum $\mathbb{G}=(G, g, \chi, \mu)$ is a group datum with $\mu=0$, with $d=o(\chi(g))<o(g)$, with $\chi^{d} \neq 1$, and such that there does not exist $\sigma \in Z^{2}(G, \dot{k})$ with $\chi^{d}(h) \sigma\left(h, g^{d}\right)=\sigma\left(g^{d}, h\right), \forall h \in G$. In this case we simply write $\mathbb{G}=(G, g, \chi)$.

- A type $\mathbf{V}$ group datum $\mathbb{G}=(G, g, \chi, \mu)$ is a group datum with $\mu=0$, $d=o(\chi(g))<o(g)$ with $\chi^{d} \neq 1$, and such that there exists $\sigma \in Z^{2}(G, \dot{k})$ with $\chi^{d}(h) \sigma\left(h, g^{d}\right)=\sigma\left(g^{d}, h\right), \forall h \in G$. In this case we simply write $\mathbb{G}=(G, g, \chi)$.

- A type VI group datum $\mathbb{G}=(G, g, \chi, \mu)$ is a group datum with $\mu \neq 0$ (and hence $d=o(\chi(g))<o(g)$ and $\left.\chi^{o(\chi(g))}=1\right)$.

Let $\mathbb{G}=(G, g, \chi, \mu)$ be a group datum. A Hopf algebra $A(\mathbb{G})$ is associated with $\mathbb{G}$ in [6]. We will slightly change the conventions of [6] for the formula defining the coproduct, but this will not change the whole set of isomorphism classes. As an algebra $A(\mathbb{G})$ is the quotient of the free product algebra $k[x] * k[G]$ by the two-sided ideal generated by the relations

$$
x h=\chi(h) h x, \forall h \in H, \quad x^{d}=\mu\left(1-g^{d}\right), \text { where } d=o(\chi(g)) .
$$

The Hopf algebra structure of $A(\mathbb{G})$ is defined by:

$$
\begin{aligned}
& \Delta(x)=1 \otimes x+x \otimes g, \quad \varepsilon(x)=0, \quad S(x)=-x g^{-1}, \\
& \Delta(h)=h \otimes h, \quad \varepsilon(h)=1, \quad S(h)=h^{-1}, \quad \forall h \in G .
\end{aligned}
$$

Using the diamond lemma [2], it is not difficult to see that the set $\left\{h x^{i}, 0 \leq i \leq\right.$ $d-1, h \in G\}$ is a linear basis of $A(\mathbb{G})$, and hence $\operatorname{dim}_{k}(A(\mathbb{G}))=|G| d$.

The Hopf algebras $A(\mathbb{G})$ have been shown in $[6]$ to be exactly the monomial non semisimple Hopf algebras: see [6] for the precise concept of a monomial Hopf algebra.

Several particular cases of this Hopf algebra construction were considered in the literature: the Taft algebras [17], the simple-pointed finite dimensional Hopf algebras (see [14])... See section 4. In fact a group datum $\mathbb{G}$ with $\mu=0$ is exactly a one-dimensional Yetter-Drinfeld module over the group algebra $k[G]$, and the Hopf algebra $A(\mathbb{G})$ is a Radford biproduct of the algebra $k[x] /\left(x^{d}\right)$ by $k[G]$. More generally, this construction might be done for any one-dimensional Yetter-Drinfeld module over an arbitrary Hopf algebra $H$ : see e.g. [1, 4].

Let $\mathbb{G}=(G, g, \chi, \mu)$ be a group datum. We need a few more informations concerning the Hopf algebra $A(\mathbb{G})$. The grouplike elements of $A(\mathbb{G})$ coincide with the group $G$. For $h \in G$, the set of $(1, h)$-primitives (i.e. elements $y \in A(\mathbb{G})$ such that $\Delta(y)=1 \otimes y+y \otimes h)$ is $k x \oplus k(g-1)$ if $h=g$ and $k(h-1)$ otherwise. From this we see that if $\mathbb{G}_{1}=\left(G_{1}, g_{1}, \chi_{1}, \mu_{1}\right)$ and $\mathbb{G}_{2}=\left(G_{2}, g_{2}, \chi_{2}, \mu_{2}\right)$ are group data, then the 
Hopf algebras $A\left(\mathbb{G}_{1}\right)$ and $A\left(\mathbb{G}_{2}\right)$ are isomorphic if and only if the group data are isomorphic in the sense of [6], namely there exists a group isomorphism $f: G_{1} \longrightarrow G_{2}$ such that $f\left(g_{1}\right)=g_{2}$ and $\chi_{2} \circ f=\chi_{1}$, and $\delta \in \dot{k}$ such that $\mu_{1}=\delta^{d} \mu_{2}$. We also see that if $\mathbb{G}=(G, g, \chi)$ is a group datum of type I to $\mathrm{V}$, then

$$
\operatorname{Aut}_{\text {Hopf }}(A(\mathbb{G})) \cong \operatorname{Aut}_{g, \chi}(G) \times \dot{k},
$$

where $\operatorname{Aut}_{g, \chi}(G)=\{u \in \operatorname{Aut}(G) \mid u(g)=g, \chi \circ u=\chi\}$. If $\mathbb{G}=(G, g, \chi, \mu)$ is a type VI group datum, then

$$
\operatorname{Aut}_{\text {Hopf }}(A(\mathbb{G})) \cong \operatorname{Aut}_{g, \chi}(G) \times \mu_{d}
$$

\subsection{Cohomological preliminaries}

In this subsection we introduce modified versions of the second cohomology group of a group. We will only need elementary group cohomology. In this setting, an appropriate reference is $[8$.

Let $G$ be a group and let $g \in G$ be a central element. We put

$$
\begin{gathered}
Z_{g}^{2}(G, \dot{k})=\left\{\sigma \in Z^{2}(G, \dot{k}), \sigma(g, h)=\sigma(h, g), \forall h \in G\right\} \quad \text { and } \\
B_{g}^{2}(G, \dot{k})=\{\partial(\mu), \mu: G \rightarrow \dot{k}, \mu(g)=1=\mu(1)\}
\end{gathered}
$$

For $g_{1}, g_{2} \in Z(G)$, it is clear that $B_{g_{2}}^{2}(G, \dot{k})$ is a subgroup of $Z_{g_{1}}^{2}(G, \dot{k})$ and we define

$$
H_{g_{1}, g_{2}}^{2}(G, \dot{k})=Z_{g_{1}}^{2}(G, \dot{k}) / B_{g_{2}}^{2}(G, \dot{k}) .
$$

We have $H_{1,1}^{2}(G, \dot{k})=H^{2}(G, \dot{k})$. These modified second cohomology groups will be useful for the description of Galois objects and biGalois groups over the non semisimple monomial Hopf algebras. Some explicit computations will be done in Section 4.

Here is an another useful construction. Let $G$ be a group and let $g \in Z(G)$ be an element of order $n$. Consider the group morphism

$$
\epsilon_{0}: Z^{2}(G, \dot{k}) \longrightarrow \dot{k}, \quad \sigma \longmapsto \sigma(g, g) \ldots \sigma\left(g, g^{n-1}\right) .
$$

It is clear that $\epsilon_{0}$ induces a group morphism

$$
\epsilon: H_{1, g}^{2}(G, \dot{k}) \longrightarrow \dot{k} .
$$

In this way we have an action by automorphism of $H_{1, g}^{2}(G, \dot{k})$ on the additive group $(k,+)$, and we can form the semi-direct product $H_{1, g}^{2}(G, \dot{k}) \ltimes k$.

Let us close this section by a useful fact, to be used freely in the rest of the paper. Let $G$ be a group, let $H \subset G$ be a central subgroup and let $\sigma \in Z^{2}(G, \dot{k})$. Then the map

$$
B_{\sigma}: G \times H \longrightarrow \dot{k},(g, h) \longmapsto \sigma(g, h) \sigma(h, g)^{-1},
$$

is a pairing between the groups $H$ and $G$ (see [8], Lemma 2.2.6). 


\section{Description of Galois objects}

In this section we determine the Galois objects for the Hopf algebra associated to a group datum. We only consider group data of type I,II, III, IV or V. The type VI case will be treated thanks to the results in the next section, and may be reduced to the type III case: see Corollary 3.18. Let us state the main result, for which the cases of type I, II, III, IV and V will be proved in this section.

Theorem 2.1 Let $\mathbb{G}=(G, g, \chi, \mu)$ be a group datum. Then according to the type of $\mathbb{G}$, we have the following description for $\operatorname{Gal}(A(\mathbb{G}))$.

- Type I: $\operatorname{Gal}(A(\mathbb{G})) \cong H^{2}(G, \dot{k}) \times k$.

- Type II and IV: $\operatorname{Gal}(A(\mathbb{G})) \cong H^{2}(G, \dot{k})$.

- Type III, V and VI: $\operatorname{Gal}(A(\mathbb{G})) \cong H^{2}(G, \dot{k}) \amalg H_{g^{d}, g^{d}}^{2}(G, \dot{k})$.

The explicit bijections will be constructed during the proof. Most of the proof will be done without any assumption on the type of the group datum. Case by case arguments will be used only at the very end.

We fix a group datum $\mathbb{G}=(G, g, \chi)$, with as usual $d=o(\chi(g))>1$. The key construction is the following one.

Definition 2.2 For $\sigma \in Z^{2}(G, \dot{k})$ and $a \in k$, we define the algebra $A_{\sigma, a}(\mathbb{G})$ to be the algebra presented by generators $X,\left(T_{h}\right)_{h \in G}$ with defining relations, $\forall h, h_{1}, h_{2} \in G$ :

$$
T_{h_{1}} T_{h_{2}}=\sigma\left(h_{1}, h_{2}\right) T_{h_{1} h_{2}}, \quad T_{1}=1, \quad X T_{h}=\chi(h) T_{h} X, \quad X^{d}=a T_{g^{d}} .
$$

Proposition 2.3 The algebra $A_{\sigma, a}(\mathbb{G})$ has a right $A(\mathbb{G})$-comodule algebra structure with coaction $\alpha: A_{\sigma, a}(\mathbb{G}) \longrightarrow A_{\sigma, a}(\mathbb{G}) \otimes A(\mathbb{G})$ defined by

$$
\alpha(X)=1 \otimes x+X \otimes g, \quad \alpha\left(T_{h}\right)=T_{h} \otimes h, \quad \forall h \in G .
$$

Then $A_{\sigma, a}(\mathbb{G})$ is a right $A(\mathbb{G})$-Galois object if and only if

$$
a \sigma\left(g^{d}, h\right)=a \chi(h)^{d} \sigma\left(h, g^{d}\right), \quad \forall h \in G .
$$

In this case the set $\left\{T_{h} X^{i}, h \in G, 0 \leq i \leq d-1\right\}$ is a linear basis of $A_{\sigma, a}(\mathbb{G})$, and the map $\Phi_{\sigma, a}: A(\mathbb{G}) \longrightarrow A_{\sigma, a}(\mathbb{G}), h x^{i} \longmapsto T_{h} X^{i}$ is a right $A(\mathbb{G})$-colinear isomorphism.

Proof. It is easy to check that the map $\alpha$ is a well-defined algebra map that endows $A_{\sigma, a}(\mathbb{G})$ with a right $A(\mathbb{G})$-comodule algebra structure. Assume that $A_{\sigma, a}(\mathbb{G})$ is a right $A(\mathbb{G})$-Galois object. Then since $A(\mathbb{G})$ is finite-dimensional, it must be cleft and $\operatorname{dim} A_{\sigma, a}(\mathbb{G})=|G| d$. The set $\left\{T_{h} X^{i}, h \in G, 0 \leq i \leq d-1\right\}$ clearly generate $A_{\sigma, a}(\mathbb{G})$ linearly, and hence must be a basis. Let $h \in G$. Then $a \sigma\left(g^{d}, h\right) T_{g^{d} h}=a T_{g^{d}} T_{h}=$ $X^{d} T_{h}=\chi(h)^{d} T_{h} X^{d}=\chi(h)^{d} a T_{h} T_{g^{d}}=\chi(h)^{d} a \sigma\left(h, g^{d}\right) T_{h g^{d}}$ and hence it follows that (2.1) holds.

Conversely assume that (2.1) holds. Endow the set $S=\left\{X, T_{h}, h \in G\right\}$ with a total order such that $T_{h}<X, \forall h \in G$. Then endow the set of monomials in elements of 
$S$ in such a way that two monomial of different length are ordered according to their length, and that two monomial of equal length are ordered lexicographically with respect to their indices. Our presentation is compatible with this order, there are three inclusion ambiguities $\left(T_{1} T_{h}, T_{1} T_{h}\right),\left(T_{h} T_{1}, T_{h} T_{1}\right)$ and $\left(X T_{1}, X T_{1}\right)$ which are clearly resolvable, and there are the following overlap ambiguities: $\left(T_{h_{1}} T_{1}, T_{1}\right),\left(T_{1}, T_{1} T_{h_{2}}\right)$, $\left(T_{h_{1}} T_{h_{2}}, T_{h_{2}} T_{h_{1}}\right),\left(X T_{h}, T_{h} T_{h_{2}}\right),\left(X T_{1}, T_{1}\right),\left(X^{i} X^{d-i}, X^{d-i} X^{i}\right),\left(X^{d}, X T_{h}\right)$. These ambiguities are easily seen to be resolvable, using (2.1) for the last one. We can use the diamond lemma [2] to conclude that $\left\{T_{h} X^{i}, h \in G, 0 \leq i \leq d-1\right\}$ is a linear basis of $A_{\sigma, a}(\mathbb{G})$. It is clear that the map $\Phi_{\sigma, a}$ is a right $A(\mathbb{G})$-colinear isomorphism. For $h \in G$, we have

$\kappa_{r}\left(\sigma\left(h^{-1}, h\right)^{-1} T_{h^{-1}} \otimes T_{h}\right)=1 \otimes h \quad$ and $\quad \kappa_{r}\left(1 \otimes X-\sigma\left(g^{-1}, g\right)^{-1} X T_{g^{-1}} \otimes T_{g}\right)=1 \otimes x$

and since $x$ and the elements $h \in G$ generate $A(\mathbb{G})$ as an algebra, it is easy to see that $\kappa_{r}$ is surjective. Hence $\kappa_{r}$ is an isomorphism and $A_{\sigma, a}(\mathbb{G})$ is $A(\mathbb{G})$-Galois.

We want to prove that any right $A(\mathbb{G})$-Galois object arises from the construction of Proposition 2.3. For this we need a slightly more general construction. Let $\sigma \in Z^{2}(G, \dot{k})$, let $a \in k$ and let $\psi: G \longrightarrow k$. We define the algebra $A_{\sigma, a, \psi}(\mathbb{G})$ to be the algebra presented by generators $X,\left(T_{h}\right)_{h \in G}$ with defining relations, $\forall h, h_{1}, h_{2} \in G$ :

$$
T_{h_{1}} T_{h_{2}}=\sigma\left(h_{1}, h_{2}\right) T_{h_{1} h_{2}}, \quad T_{1}=1, \quad X T_{h}=\chi(h) T_{h} X+\psi(h) T_{g h}, \quad X^{d}=a T_{g^{d}} .
$$

Lemma 2.4 The algebra $A_{\sigma, a, \psi}(\mathbb{G})$ has a right $A(\mathbb{G})$-comodule algebra structure with coaction $\alpha: A_{\sigma, a, \psi}(\mathbb{G}) \longrightarrow A_{\sigma, a, \psi}(\mathbb{G}) \otimes A(\mathbb{G})$ defined by

$$
\alpha(X)=1 \otimes x+X \otimes g, \quad \alpha\left(T_{h}\right)=T_{h} \otimes h, \quad \forall h \in G .
$$

Moreover the following assertions are equivalent.

1) $A_{\sigma, a, \psi}(\mathbb{G})$ is a right $A(\mathbb{G})$-Galois object.

2) $\operatorname{dim} A_{\sigma, a, \psi}(\mathbb{G})=|G| d$ and the set $\left\{T_{h} X^{i}, h \in G, 0 \leq i \leq d-1\right\}$ is a linear basis.

3) $A_{\sigma, a, \psi}(\mathbb{G})$ is a non-zero algebra.

Proof. It is easy to check that the map $\alpha$ is a well-defined algebra map that endows $A_{\sigma, a, \psi}(\mathbb{G})$ with a right $A(\mathbb{G})$-comodule algebra structure. The proof of 1$) \Rightarrow 2$ ) is similar to the one in Proposition 2.3 and 2$) \Rightarrow 3$ ) is obvious. Similarly to Proposition 2.3 the map $\kappa_{r}$ is surjective and if $A_{\sigma, a, \psi}(\mathbb{G})$ is a non-zero algebra we have $\operatorname{dim} A_{\sigma, a, \psi}(\mathbb{G}) \geq|G| d$, hence $\operatorname{dim} A_{\sigma, a, \psi}(\mathbb{G})=|G| d$ and we conclude that $\kappa_{r}$ is an isomorphism.

The following result motivates the introduction of the algebras $A_{\sigma, a, \psi}(\mathbb{G})$.

Lemma 2.5 Let $T$ be a right $A(\mathbb{G})$-Galois object. Then there exists $\sigma \in Z^{2}(G, \dot{k})$, $a \in k$ and $\psi: G \longrightarrow k$ such that $T \cong A_{\sigma, a, \psi}(\mathbb{G})$ as right $A(\mathbb{G})$-comodule algebras.

Proof. Let $\sigma: A(\mathbb{G}) \otimes A(\mathbb{G}) \longrightarrow k$ be a 2-cocycle such that there exists an isomorphism of $A(\mathbb{G})$-comodule algebras $f:{ }_{\sigma} A(\mathbb{G}) \longrightarrow T$. We put $X=f(x)$ and $T_{h}=f(h)$ 
for $h \in G$. It is clear that for $h_{1}, h_{2} \in G$, we have $T_{h_{1}} T_{h_{2}}=\sigma\left(h_{1}, h_{2}\right) T_{h_{1} h_{2}}$. Let $\beta$ be the coaction of $A(\mathbb{G})$ on $T$. We have

$$
\beta\left(T_{h}\right)=T_{h} \otimes h, \forall h \in G \text { and } \beta(X)=1 \otimes x+X \otimes g .
$$

Hence for $h \in G$, we have

$$
\beta\left(X T_{h}-\chi(h) T_{h} X\right)=\left(X T_{h}-\chi(h) T_{h} X\right) \otimes h g,
$$

and thus there exists $\psi(h) \in k$ such that $X T_{h}-\chi(h) T_{h} X=\psi(h) T_{g h}$. This defines a map $\psi: G \rightarrow k$. Similarly $\beta\left(X^{d}\right)=(1 \otimes x+X \otimes g)^{d}=X^{d} \otimes g^{d}$ and hence there exists $a \in k$ such that $X^{d}=a T_{g^{d}}$. In this way we have a surjective right $A(\mathbb{G})$-comodule algebras morphism $A_{\sigma, a, \psi}(\mathbb{G}) \longrightarrow T$ where $\sigma$ is the restriction of $\sigma$ to $G$. Hence $A_{\sigma, a, \psi}(\mathbb{G})$ is a non-zero algebra, is $A(\mathbb{G})$-Galois by the previous lemma, and since $A_{\sigma, a, \psi}(\mathbb{G})$ and $T$ are both $A(\mathbb{G})$-Galois, they are isomorphic via $f$.

Therefore we need to study when the algebra $A_{\sigma, a, \psi}(\mathbb{G})$ is $A(\mathbb{G})$-Galois. The technical conditions are given in the following result.

Lemma 2.6 If $A_{\sigma, a, \psi}(\mathbb{G})$ is a right $A(\mathbb{G})$-Galois object, then for $h_{1}, h_{2}, h \in G$ we have

$$
\begin{gathered}
\psi\left(h_{1} h_{2}\right)=\sigma\left(h_{1}, h_{2}\right)^{-1}\left(\chi\left(h_{1}\right) \sigma\left(h_{1}, h_{2} g\right) \psi\left(h_{2}\right)+\sigma\left(h_{1} g, h_{2}\right) \psi\left(h_{1}\right)\right), \\
\psi(h)=\psi(g)(\sigma(g, h)-\chi(h) \sigma(h, g)) \sigma(g, g)^{-1}(1-\chi(g))^{-1}, \\
a\left(\sigma\left(g^{d}, h\right)-\chi(h)^{d} \sigma\left(h, g^{d}\right)\right)=\psi(h) \psi(g h) \ldots \psi\left(g^{d-1} h\right) .
\end{gathered}
$$

Proof. Let $h_{1}, h_{2} \in G$. We have

$$
X T_{h_{1} h_{2}}=\chi\left(h_{1} h_{2}\right) T_{h_{1} h_{2}} X+\psi\left(h_{1} h_{2}\right) T_{h_{1} h_{2} g} .
$$

On the other hand we have

$$
\begin{aligned}
& X T_{h_{1} h_{2}}=\sigma\left(h_{1}, h_{2}\right)^{-1} X T_{h_{1}} T_{h_{2}}=\sigma\left(h_{1}, h_{2}\right)^{-1}\left(\chi\left(h_{1}\right) T_{h_{1}} X+\psi\left(h_{1}\right) T_{h_{1} g}\right) T_{h_{2}}= \\
& =\sigma\left(h_{1}, h_{2}\right)^{-1}\left(\chi\left(h_{1}\right) \chi\left(h_{2}\right) T_{h_{1}} T_{h_{2}} X+\chi\left(h_{1}\right) \psi\left(h_{2}\right) T_{h_{1}} T_{h_{2} g}+\psi\left(h_{1}\right) T_{h_{1} g} T_{h_{2}}\right)= \\
& =\chi\left(h_{1} h_{2}\right) T_{h_{1} h_{2}} X+\sigma\left(h_{1}, h_{2}\right)^{-1}\left(\chi\left(h_{1}\right) \psi\left(h_{2}\right) \sigma\left(h_{1}, h_{2} g\right)+\psi\left(h_{1}\right) \sigma\left(h_{1} g, h_{2}\right)\right) T_{h_{1} h_{2} g} .
\end{aligned}
$$

Hence by Lemma 2.3, Equation (2.2) holds.

Let $h \in G$. Since $g$ is central in $G$, we have $\psi(h g)=\psi(g h)$, and it is a straightforward computation to check that Equation (2.3) holds, using (2.2) and the fact that $\sigma$ is a 2-cocycle.

For $n \in \mathbb{N}^{*}$, there exists $a_{1}, \ldots a_{n-1} \in k$ such that

$$
X^{n} T_{h}=\chi(h)^{n} T_{h} X^{n}+\sum_{l=1}^{n-1} a_{l} T_{g^{l} h} X^{n-l}+\psi(h) \psi(g h) \ldots \psi\left(g^{n-1} h\right) T_{g^{n} h} .
$$

This is proved easily by induction. For $n=d$, we see, using Lemma 2.3, that Equation (2.4) holds. 
Lemma 2.7 Let $\sigma \in Z^{2}(G, \dot{k})$. Then for $h \in G$, we have

$$
\prod_{i=0}^{d-1}\left(\sigma\left(g, g^{i} h\right)-\chi\left(g^{i} h\right) \sigma\left(g^{i} h, g\right)\right)=\sigma(g, g) \ldots \sigma\left(g, g^{d-1}\right)\left(\sigma\left(g^{d}, h\right)-\chi(h)^{d} \sigma\left(h, g^{d}\right)\right) \text {. }
$$

Proof. Consider the polynomials

$$
\begin{gathered}
P(X)=\prod_{i=0}^{d-1}\left(\sigma\left(g, g^{i} h\right)-\chi\left(g^{i}\right) \sigma\left(g^{i} h, g\right) X\right) \in k[X] \quad \text { and } \\
Q(X)=\sigma(g, g) \ldots \sigma\left(g, g^{d-1}\right)\left(\sigma\left(g^{d}, h\right)-\sigma\left(h, g^{d}\right) X^{d}\right) \in k[X] .
\end{gathered}
$$

Since $\sigma$ is a 2-cocycle and since $g$ is central, we have for $i \in \mathbb{N}$ :

$$
\sigma(g, h) \sigma(h, g)^{-1}=\sigma\left(g, g^{i} h\right) \sigma\left(g^{i} h, g\right)^{-1} \text { and }\left(\sigma(g, h) \sigma(h, g)^{-1}\right)^{i}=\sigma\left(g^{i}, h\right) \sigma\left(h, g^{i}\right)^{-1} .
$$

Let $t$ be a root of $P$ : this means that there exists $i \in\{0, \ldots, d-1\}$ such that $t=$ $\sigma\left(g, g^{i} h\right) \sigma\left(g^{i} h, g\right)^{-1} \chi(g)^{-i}=\sigma(g, h) \sigma(h, g)^{-1} \chi(g)^{-i}$. Then $t^{d}=\left(\sigma(g, h) \sigma(h, g)^{-1}\right)^{d}=$ $\sigma\left(g^{d}, h\right) \sigma\left(h, g^{d}\right)^{-1}$. Hence $t$ is a root of $Q$. Conversely assume that $t$ is a root of $Q$. Then we have $t^{d} \sigma\left(g^{d}, h\right)^{-1} \sigma\left(h, g^{d}\right)=1=\left(t \sigma(g, h)^{-1} \sigma(h, g)\right)^{d}$. Hence there exists $i \in\{0, \ldots, d-1\}$ such that $t=\chi(g)^{-i} \sigma(g, h) \sigma(h, g)^{-1}=\chi(g)^{-i} \sigma\left(g, g^{i} h\right) \sigma\left(g^{i} h, g\right)^{-1}$ and $t$ is a root of $P$. It is easy to see that $P$ and $Q$ have the same constant term, and we conclude that $P=Q$. In particular $P(\chi(h))=Q(\chi(h))$ : this proves the lemma.

Lemma 2.8 Let $\sigma \in Z^{2}(G, \dot{k}), a \in k$ and $\psi: G \rightarrow k$ be such that $A_{\sigma, a, \psi}(\mathbb{G})$ is $A(\mathbb{G})$-Galois. Then there exists $a^{\prime} \in k$ such that $A_{\sigma, a, \psi}(\mathbb{G}) \cong A_{\sigma, a^{\prime}}(\mathbb{G})$ as right $A(\mathbb{G})$-comodule algebras.

Proof. We put

$$
a^{\prime}=a-\psi(g)^{d} \sigma(g, g)^{-d}(1-\chi(g))^{-d} \sigma(g, g) \ldots \sigma\left(g, g^{d-1}\right) .
$$

Let us first show that $A_{\sigma, a^{\prime}}$ is $A(\mathbb{G})$-Galois. Let $h \in G$. We have

$$
\begin{aligned}
& a^{\prime}\left(\sigma\left(g^{d}, h\right)-\chi(h)^{d} \sigma\left(h, g^{d}\right)\right)=a\left(\sigma\left(g^{d}, h\right)-\chi(h)^{d} \sigma\left(h, g^{d}\right)\right) \\
& -\psi(g)^{d} \sigma(g, g)^{-d}(1-\chi(g))^{-d} \sigma(g, g) \ldots \sigma\left(g, g^{d-1}\right)\left(\sigma\left(g^{d}, h\right)-\chi(h)^{d} \sigma\left(h, g^{d}\right)\right) \\
& =\psi(h) \psi(g h) \ldots \psi\left(g^{d-1} h\right)(\text { by Lemma } 2.6)- \\
& \psi(g)^{d} \sigma(g, g)^{-d}(1-\chi(g))^{-d} \sigma(g, g) \ldots \sigma\left(g, g^{d-1}\right)\left(\sigma\left(g^{d}, h\right)-\chi(h)^{d} \sigma\left(h, g^{d}\right)\right) \\
& =\psi(g)^{d} \sigma(g, g)^{-d}(1-\chi(g))^{-d} \\
& {\left[\left(\prod_{i=0}^{d-1}\left(\sigma\left(g, g^{i} h\right)-\chi\left(g^{i} h\right) \sigma\left(g^{i} h, g\right)\right)-\sigma(g, g) \ldots \sigma\left(g, g^{d-1}\right)\left(\sigma\left(g^{d}, h\right)-\chi(h)^{d} \sigma\left(h, g^{d}\right)\right)\right]\right.}
\end{aligned}
$$

(by Lemma 2.6) $=0$ (by Lemma 2.7). 
Hence $A_{\sigma, a^{\prime}}(\mathbb{G})$ is $A(\mathbb{G})$-Galois by Proposition 2.3. Now put $\lambda=\psi(g) \sigma(g, g)^{-1}(1-$ $\chi(g))^{-1}$. It is straightforward to check, using Lemma 2.6, that there exists an $A(\mathbb{G})$ comodule algebra morphism $f: A_{\sigma, a, \psi}(\mathbb{G}) \longrightarrow A_{\sigma, a^{\prime}}(\mathbb{G})$ such that $f(X)=X+\lambda T_{g}$ and $f\left(T_{h}\right)=T_{h}, \forall h \in G$. Then $f$ is an isomorphism since $A_{\sigma, a, \psi}(\mathbb{G})$ and $A_{\sigma, a^{\prime}}(\mathbb{G})$ are $A(\mathbb{G})$-Galois.

Combining Lemmas 2.5 and 2.8, we get the following result.

Proposition 2.9 Let $T$ be a right $A(\mathbb{G})$-Galois object. Then there exists $\sigma \in Z^{2}(G, \dot{k})$ and $a \in k$ such that $T \cong A_{\sigma, a}(\mathbb{G})$ as right $A(\mathbb{G})$-comodule algebras.

The next step is to classify the Galois objects $A_{\sigma, a}(\mathbb{G})$.

Proposition 2.10 Let $\sigma, \tau \in Z^{2}(G, \dot{k})$ and $a, b \in k$ be such that $A_{\sigma, a}(\mathbb{G})$ and $A_{\tau, b}(\mathbb{G})$ are $A(\mathbb{G})$-Galois objects. Then the right $A(\mathbb{G})$-comodule algebras $A_{\sigma, a}(\mathbb{G})$ and $A_{\tau, b}(\mathbb{G})$ are isomorphic if and only if there exists $\mu: G \longrightarrow \dot{k}$ with $\mu(1)=1$ such that

$$
\sigma=\partial(\mu) \tau \quad \text { and } \quad b=a \mu\left(g^{d}\right)
$$

Proof. Let $f: A_{\sigma, a}(\mathbb{G}) \longrightarrow A_{\tau, b}(\mathbb{G})$ be an $A(\mathbb{G})$-colinear algebra isomorphism. Then $\Phi_{\tau, b}^{-1} \circ f \circ \Phi_{\sigma, a}$ is a right $A(\mathbb{G})$-colinear automorphism of $A(\mathbb{G})$, and hence there exists a convolution invertible linear map $\phi: A(\mathbb{G}) \rightarrow k$ such that $\Phi_{\tau, b}^{-1} \circ f \circ \Phi_{\sigma, a}=\phi *$ id. From this we see easily see that there exists $\mu: G \longrightarrow \dot{k}$ and $\lambda \in k$ such that

$$
f(X)=X+\lambda T_{g} \quad \text { and } \quad f\left(T_{h}\right)=\mu(h) T_{h}, \forall h \in G .
$$

It is then clear that $\sigma=\partial(\mu) \tau$ and that $\mu(1)=1$. We have

$$
\begin{gathered}
f\left(X T_{g}\right)=\mu(g) X T_{g}+\lambda \mu(g) \tau(g, g) T_{g^{2}} \text { and } \\
f\left(\chi(g) T_{g} X\right)=\chi(g) \mu(g) T_{g} X+\chi(g) \lambda \tau(g, g) \mu(g) T_{g^{2}} .
\end{gathered}
$$

Since $\chi(g) \neq 1$, we have $\lambda=0$. Then $b T_{g^{d}}=X^{d}=f\left(X^{d}\right)=\mu\left(g^{d}\right) a T_{g^{d}}$ and hence $b=a \mu\left(g^{d}\right)$.

Conversely if $\sigma=\partial(\mu) \tau$ with $\mu(1)=1$ and $b=a \mu\left(g^{d}\right)$, it is straightforward to check that there exists a right $A(\mathbb{G})$-comodule algebra isomorphism $f: A_{\sigma, a}(\mathbb{G}) \rightarrow$ $A_{\tau, b}(\mathbb{G})$ such that $f(X)=X$ and $f\left(T_{h}\right)=\mu(h) T_{h}, \forall h \in G$.

We can now finish the proof of Theorem 2.1 with case by case arguments.

Proposition 2.11 Let $\mathbb{G}=(G, g, \chi)$ be a type I group datum. For any $\sigma \in Z^{2}(G, \dot{k})$ and $a \in k$, then $A_{\sigma, a}(\mathbb{G})$ is $A(\mathbb{G})$-Galois. The map $Z^{2}(G, \dot{k}) \times k \longrightarrow \operatorname{Gal}(A(\mathbb{G}))$, $(\sigma, a) \longmapsto\left[A_{\sigma, a}(\mathbb{G})\right]$, induces a bijection

$$
H^{2}(G, \dot{k}) \times k \cong \operatorname{Gal}(A(\mathbb{G})) .
$$


Proof. It is clear that for any $\sigma \in Z^{2}(G, \dot{k})$ and $a \in k$, condition (2.1) is satisfied and hence $A_{\sigma, a}(\mathbb{G})$ is $A(\mathbb{G})$-Galois by Proposition 2.3. The map $Z^{2}(G, \dot{k}) \times k \longrightarrow$ $\operatorname{Gal}(A(\mathbb{G})),(\sigma, a) \longmapsto\left[A_{\sigma, a}(\mathbb{G})\right]$, induces, by Proposition 2.10, an injective map $H^{2}(G, \dot{k}) \times k \longrightarrow \operatorname{Gal}(A(\mathbb{G}))$. This map is surjective by Proposition 2.9: this concludes the proof.

Proposition 2.12 Let $\mathbb{G}=(G, g, \chi)$ be a type II group datum. For any $\sigma \in$ $Z^{2}(G, \dot{k})$, then $A_{\sigma, 0}(\mathbb{G})$ is $A(\mathbb{G})$-Galois. The map $Z^{2}(G, \dot{k}) \longrightarrow \operatorname{Gal}(A(\mathbb{G})), \sigma \longmapsto$ $\left[A_{\sigma, 0}(\mathbb{G})\right]$, induces a bijection

$$
H^{2}(G, \dot{k}) \cong \operatorname{Gal}(A(\mathbb{G})) .
$$

Proof. For any $\sigma \in Z^{2}(G, \dot{k})$ and $a=0$, condition (2.1) is satisfied and hence $A_{\sigma, 0}(\mathbb{G})$ is $A(\mathbb{G})$-Galois by Proposition 2.3. The map $Z^{2}(G, \dot{k}) \longrightarrow \operatorname{Gal}(A(\mathbb{G})), \sigma \longmapsto$ $\left[A_{\sigma, 0}(\mathbb{G})\right]$, induces, by Proposition 2.10, an injective map $H^{2}(G, \dot{k}) \longrightarrow \operatorname{Gal}(A(\mathbb{G}))$. Let $T$ be a right $A(\mathbb{G})$-Galois object: by Proposition 2.10 there exists $\sigma \in Z^{2}(G, \dot{k})$ and $a \in k$ such that $T \cong A_{\sigma, a}(\mathbb{G})$. But since $\chi^{d} \neq 1$, condition (2.1) is satisfied only if $a=0$. This concludes the proof.

Proposition 2.13 Let $\mathbb{G}=(G, g, \chi)$ be a type III group datum. Then the maps $Z^{2}(G, \dot{k}) \longrightarrow \operatorname{Gal}(A(\mathbb{G})), \sigma \longmapsto\left[A_{\sigma, 0}(\mathbb{G})\right]$, and $Z_{g^{d}}^{2}(G, \dot{k}) \longrightarrow \operatorname{Gal}(A(\mathbb{G}))$, $\sigma \longmapsto\left[A_{\sigma, 1}(\mathbb{G})\right]$, induce a bijection

$$
H^{2}(G, \dot{k}) \amalg H_{g^{d}, g^{d}}^{2}(G, \dot{k}) \cong \operatorname{Gal}(A(\mathbb{G})) .
$$

Proof. Our two maps induce a map $H^{2}(G, \dot{k}) \amalg H_{g^{d}, g^{d}}^{2}(G, \dot{k}) \longrightarrow \operatorname{Gal}(A(\mathbb{G}))$, which is injective by Proposition 2.10. Let $T$ be a right $A(\mathbb{G})$-Galois object: by Proposition 2.9 there exists $\sigma \in Z^{2}(G, \dot{k})$ and $a \in k$ such that $T \cong A_{\sigma, a}(\mathbb{G})$. If $a \neq 0$, then $\sigma \in Z_{g^{d}}^{2}(G, \dot{k})$ by Proposition 2.3. Consider $\mu: G \longrightarrow \dot{k}$ such that $\mu(1)=1$ and $\mu\left(g^{d}\right)=a$. Then $A_{\sigma, a}(\mathbb{G}) \cong A_{\partial(\mu) \sigma, 1}(\mathbb{G})$ by Proposition 2.10, and hence our map is also surjective.

The proofs of the type IV and V cases are similar and left to the reader, and conclude the proof of Theorem 2.1 (up to the type VI case).

Proposition 2.14 Let $\mathbb{G}=(G, g, \chi)$ be a type IV group datum. Then the map $Z^{2}(G, \dot{k}) \longrightarrow \operatorname{Gal}(A(\mathbb{G})), \sigma \longmapsto\left[A_{\sigma, 0}(\mathbb{G})\right]$, induces a bijection

$$
H^{2}(G, \dot{k}) \cong \operatorname{Gal}(A(\mathbb{G})) \text {. }
$$

Proposition 2.15 Let $\mathbb{G}=(G, g, \chi)$ be a type $V$ group datum. Let $\tau \in Z^{2}(G, \dot{k})$ be such that $\chi(h)^{d}=\tau\left(g^{d}, h\right) \tau\left(h, g^{d}\right)^{-1}, \forall h \in G$. Then $\forall \sigma \in Z_{g^{d}}^{2}(G, \dot{k}), A_{\tau \sigma, 1}(\mathbb{G})$ is $A(\mathbb{G})$-Galois. $\quad$ The maps $Z^{2}(G, \dot{k}) \longrightarrow \operatorname{Gal}(A(\mathbb{G})), \sigma \longmapsto\left[A_{\sigma, 0}(\mathbb{G})\right]$, and $Z_{g^{d}}^{2}(G, \dot{k}) \longrightarrow \operatorname{Gal}(A(\mathbb{G})), \sigma \longmapsto\left[A_{\tau \sigma, 1}(\mathbb{G})\right]$, induce a bijection

$$
H^{2}(G, \dot{k}) \amalg H_{g^{d}, g^{d}}^{2}(G, \dot{k}) \cong \operatorname{Gal}(A(\mathbb{G})) .
$$


We conclude the section with a few words concerning the notion of homotopy equivalence of Hopf-Galois extensions, recently introduced by Kassel and Schneider 10. Let $\mathbb{G}=(G, g, \chi, \mu)$ be a group datum and let $\mathcal{H}_{k}(A(\mathbb{G}))$ be the set of homotopy classes of right $A(\mathbb{G})$-Galois extensions of $k$ (see [10] for the precise definition). Then we have

$$
\mathcal{H}_{k}(A(\mathbb{G})) \cong H^{2}(G, \dot{k}) .
$$

Indeed if $\mu=0$, then $A(\mathbb{G})=\oplus_{i=0}^{d-1} k[G] x^{i}$ and hence $A(\mathbb{G})$ is an $\mathbb{N}$-graded Hopf algebra. Therefore combining Corollary 2.9 and Proposition 3.2 of [10], we have the claimed result. Then one gets the result in the type VI case by combining Corollary 2.11 of [10] and Corollary 3.18 in the next section.

\section{$3 \quad$ BiGalois groups}

In this section we give a general description of the biGalois group of a monomial Hopf algebra. Several particular cases will be studied in more detail in the next section.

Just like in the previous section, our first goal is to state our main result. We first need to introduce some terminology. Let $\mathbb{G}=(G, g, \chi, \mu)$ be a group datum. We consider the subgroup $\operatorname{Aut}_{g}(G)$ of elements $u \in \operatorname{Aut}(G)$ satisfying $u(g)=g$ and the modified cohomology group $H_{1, g}^{2}(G, \dot{k})=Z^{2}(G, \dot{k}) / B_{g}^{2}(G, \dot{k})$ of the first section. The $\operatorname{group} \operatorname{Aut}_{g}(G)$ has a natural right action by automorphisms on $H_{1, g}^{2}(G, \dot{k})$, and hence we may form the semi-direct product $\operatorname{Aut}_{g}(G) \ltimes H_{1, g}^{2}(G, \dot{k})$. We define now

$\Gamma(\mathbb{G})=\left\{(u, \bar{\sigma}) \in \operatorname{Aut}_{g}(G) \times H_{1, g}^{2}(G, \dot{k}), \chi \circ u(h)=\sigma(g, h)^{-1} \sigma(h, g) \chi(h), \forall h \in G\right\}$.

It is clear that, since $g$ is central in $G$, that the defining equation for the elements of $\Gamma(\mathbb{G})$ does not depend of the choice of a representant of a (modified) cohomology class. The following lemma is a straightforward verification.

Lemma 3.1 Let $\mathbb{G}=(G, g, \chi, \mu)$ be a group datum. Then $\Gamma(\mathbb{G})$ is a subgroup of $\operatorname{Aut}_{g}(G) \ltimes H_{1, g}^{2}(G, \dot{k})$.

The group morphism $\epsilon: H_{1, g}^{2}(G, \dot{k}) \longrightarrow \dot{k}$ has a natural continuation to $\Gamma(\mathbb{G})$, and hence we may form the semi-direct product $\Gamma(\mathbb{G}) \ltimes k$. We have now all the ingredients to state the main result of the section.

Theorem 3.2 Let $\mathbb{G}=(G, g, \chi)$ be a group datum. Then according to the type of $\mathbb{G}$, we have the following group isomorphism.

- Type $I$ : $\operatorname{BiGal}(A(\mathbb{G})) \cong \Gamma(\mathbb{G}) \ltimes k$.

- Type II, III, IV, V and VI: $\operatorname{BiGal}(A(\mathbb{G})) \cong \Gamma(\mathbb{G})$.

Types V and VI will be shown to reduce to type III. Similarly to the previous section, we begin with general results and constructions. 
Proposition 3.3 Let $\mathbb{G}=(G, g, \chi)$ be a group datum. Let $\sigma \in Z^{2}(G, \dot{k})$ and $a \in k$ with $a=0$ if $\mathbb{G}$ is not of type $I: A_{\sigma, a}(\mathbb{G})$ is $A(\mathbb{G})$-Galois. Assume that there exists $u \in \operatorname{Aut}_{g}(G)$ such that $(u, \bar{\sigma}) \in \Gamma(\mathbb{G})$. Then $A_{\sigma, a}(\mathbb{G})$ has a left $A(\mathbb{G})$-comodule algebra structure $\beta_{u}: A_{\sigma, a}(\mathbb{G}) \longrightarrow A(\mathbb{G}) \otimes A_{\sigma, a}(\mathbb{G})$ defined by

$$
\beta_{u}(X)=1 \otimes X+x \otimes T_{g}, \quad \beta_{u}\left(T_{h}\right)=u(h) \otimes T_{h}, \forall h \in G .
$$

Furthermore $A_{\sigma, a}(\mathbb{G})$ is an $A(\mathbb{G})-A(\mathbb{G})$-bicomodule algebra and is $A(\mathbb{G})$-biGalois. This Hopf biGalois extension is denoted by $A_{\sigma, a}^{u}(\mathbb{G})$.

Proof. It is easy to check that $\beta_{u}$ is a well-defined algebra map, using that $(u, \bar{\sigma}) \in$ $\Gamma(\mathbb{G})$ when checking that $\beta_{u}\left(X T_{h}\right)=\beta_{u}\left(\chi(h) T_{h} X\right)$, that endows $A_{\sigma, a}(\mathbb{G})$ with an $A(\mathbb{G})$ - $A(\mathbb{G})$-bicomodule structure. There remains to check that $\kappa_{l}: A_{\sigma, a}(\mathbb{G}) \otimes$ $A_{\sigma, a}(\mathbb{G}) \longrightarrow A(\mathbb{G}) \otimes A_{\sigma, a}(\mathbb{G})$ is bijective. We have $\kappa_{l}\left(\sigma\left(g, g^{-1}\right)^{-1}\left(X \otimes T_{g^{-1}}-1 \otimes\right.\right.$ $\left.\left.X T_{g^{-1}}\right)\right)=x \otimes 1$ and $\kappa_{l}\left(\sigma\left(h, h^{-1}\right)^{-1} T_{h} \otimes T_{h^{-1}}\right)=u(h) \otimes 1$ for $h \in G$. Since the elements $x$ and $u(h), h \in G$ generate $A(\mathbb{G})$ as an algebra and since $\kappa_{l}$ is right $A_{\sigma, a}(\mathbb{G})$-linear, we conclude that it is surjective and an isomorphism.

The next step is to classify the Hopf biGalois extensions just constructed.

Proposition 3.4 Let $\mathbb{G}=(G, g, \chi)$ be a group datum. Let $\sigma, \tau \in Z^{2}(G, \dot{k})$ and $a, b \in k$ with $a=b=0$ if $\mathbb{G}$ is not of type $I: A_{\sigma, a}(\mathbb{G})$ and $A_{\tau, b}(\mathbb{G})$ are $A(\mathbb{G})$-Galois. Assume that there exist $u, v \in \operatorname{Aut}_{g}(G)$ such that $(u, \bar{\sigma}) \in \Gamma(\mathbb{G})$ and $(v, \bar{\tau}) \in \Gamma(\mathbb{G})$. Then the $A(\mathbb{G})$-biGalois extensions $A_{\sigma, a}^{u}(\mathbb{G})$ and $A_{\tau, b}^{v}(\mathbb{G})$ are isomorphic if and only if $u=v$ and there exists $\mu: G \longrightarrow \dot{k}$ satisfying $\mu(1)=\mu(g)=1, \sigma=\partial(\mu) \tau$ and $b=a \mu\left(g^{d}\right)$.

Proof. Let $f: A_{\sigma, a}^{u}(\mathbb{G}) \longrightarrow A_{\tau, b}^{v}(\mathbb{G})$ be an $A(\mathbb{G})$-bicolinear isomorphism. By Proposition 2.10 and its proof, there exists $\mu: G \longrightarrow \dot{k}$ with $\mu(g)=1, \sigma=\partial(\mu) \tau$, $b=a \mu\left(g^{d}\right)$, such that $f(X)=X$ and $f\left(T_{h}\right)=\mu(h) T_{h}$ for $h \in G$. Using that $f$ is left colinear, we see that $u=v$ and that $\mu(g)=1$. The converse assertion is clear from the above considerations.

We now connect the group structures of $\Gamma(\mathbb{G})$ and $\operatorname{BiGal}(A(\mathbb{G}))$.

Proposition 3.5 Let $\mathbb{G}=(G, g, \chi)$ be a group datum. Let $\sigma, \tau \in Z^{2}(G, \dot{k})$ and $a, b \in k$ with $a=b=0$ if $\mathbb{G}$ is not of type $I: A_{\sigma, a}(\mathbb{G})$ and $A_{\tau, b}(\mathbb{G})$ are $A(\mathbb{G})$ Galois. Assume that there exist $u, v \in \operatorname{Aut}_{g}(G)$ such that $(u, \bar{\sigma}) \in \Gamma(\mathbb{G})$ and $(v, \bar{\tau}) \in$ $\Gamma(\mathbb{G})$. Put $\nu=(\sigma \circ v \times v) \tau$ and $c=\tau(g, g) \ldots \tau\left(g, g^{d-1}\right) a+b$. Then $A_{\nu, c}(\mathbb{G})$ is right $A(\mathbb{G})$-Galois, $(u \circ v, \bar{\nu}) \in \Gamma(\mathbb{G})$ and the $A(\mathbb{G})$-biGalois extensions $A_{\nu, c}^{\text {uov }}(\mathbb{G})$ and $A_{\sigma, a}^{u}(\mathbb{G}) \square_{A(\mathbb{G})} A_{\tau, b}^{v}(\mathbb{G})$ are isomorphic.

Proof. It is clear that $A_{\nu, c}(\mathbb{G})$ is $A(\mathbb{G})$-Galois. We have $(u \circ v, \bar{\nu}) \in \Gamma(\mathbb{G})$ by Lemma 3.1 and hence we can consider the $A(\mathbb{G})$-biGalois extension $A_{\nu, c}^{u \circ v}(\mathbb{G})$. It is easy to see that $1 \otimes X+X \otimes T_{g} \in A_{\sigma, a}^{u}(\mathbb{G}) \square_{A(\mathbb{G})} A_{\tau, b}^{v}(\mathbb{G})$ and that for $h \in G$, 
$T_{v(h)} \otimes T_{h} \in A_{\sigma, a}^{u}(\mathbb{G}) \square_{A(\mathbb{G})} A_{\tau, b}^{v}(\mathbb{G})$. One checks now that there exists an algebra morphism

$$
\gamma: A_{\nu, c}^{u \circ v}(\mathbb{G}) \longrightarrow A_{\sigma, a}^{u}(\mathbb{G}) \square_{A(\mathbb{G})} A_{\tau, b}^{v}(\mathbb{G})
$$

such that $\gamma(X)=1 \otimes X+X \otimes T_{g}$ and $\gamma\left(T_{h}\right)=T_{v(h)} \otimes T_{h}, \forall h \in G$. It is clear that $\gamma$ is $A(\mathbb{G})$-bicolinear, and since it is a morphism of Galois extensions, it is an isomorphism.

Now we need to show that we have constructed all the $A(\mathbb{G})$-biGalois extensions. We will need case by case arguments. We begin with a property which, unfortunately, only holds in general for type I or II group data.

Lemma 3.6 Let $\mathbb{G}=(G, g, \chi)$ be a group datum of type $I$ or II. Let $\sigma \in Z^{2}(G, \dot{k})$. Then there exists $u \in \operatorname{Aut}_{g}(G)$ such that $(u, \bar{\sigma}) \in \Gamma(\mathbb{G})$.

Proof. Since $\mathbb{G}$ is of type I or II we have $d=o(\chi(g))=o(g)$ and $\chi$ induces a group isomorphism $\langle g\rangle \cong \mu_{d}$. Let $s: \mu_{d} \longrightarrow\langle g\rangle$ be a group morphism such that $\chi \circ s=$ id. Now let $\psi: G \longrightarrow \dot{k}$ be a group morphism. Define a map $u: G \longrightarrow G$ by $u(h)=s(\psi(h)) h, \forall h \in G$. Then it is easy to see that $u$ is a group morphism $(g$ is central) and that $\chi \circ u(h)=\psi(h) \chi(h), \forall h \in G$. Furthermore $u$ is an isomorphism and $u(g)=g$ if $\psi(g)=1$. This general argument, applied to the group morphism $G \longrightarrow \mu_{d}, h \longmapsto \sigma(g, h)^{-1} \sigma(h, g)$, proves our lemma.

Combining Propositions 2.11, 2.12 and 3.3, Lemma 3.6 with Theorem 3.5 and Corollary 5.7 of [15], we get the following result, which generalizes Corollaries 4 and 18 in [16].

Corollary 3.7 Let $\mathbb{G}=(G, g, \chi)$ be a group datum of type I or II. Then any right $A(\mathbb{G})$-Galois object is $A(\mathbb{G})$-biGalois. The Hopf algebra $A(\mathbb{G})$ is categorically rigid: if $H$ is any Hopf algebra such that the monoidal $k$-linear categories of comodules of $A(\mathbb{G})$ and $H$ are equivalent, then $H \cong A(\mathbb{G})$ as Hopf algebras.

We can finish the proof of Theorem 3.2 in the case of type I and II group data.

Proposition 3.8 Let $\mathbb{G}=(G, g, \chi)$ be a type I group datum. Then we have a group isomorphism:

$$
\begin{aligned}
\Psi: \Gamma(\mathbb{G}) \ltimes k & \longrightarrow \operatorname{BiGal}(A(\mathbb{G})) \\
(u, \bar{\sigma}, a) & \longmapsto\left[A_{\sigma, a}^{u}\right] .
\end{aligned}
$$

Proof. It follows immediately from Propositions 2.11, 3.3 and 3.4 that $\Psi$ is a well-defined injective map. Also it is clear from Proposition 3.5 that $\psi$ is a group morphism. There just remains to show that $\Psi$ is surjective. Let $Z$ be an $A(\mathbb{G})$ biGalois extension. By Proposition 2.11 we can assume that $Z=A_{\sigma, a}(\mathbb{G})$ as right $A(\mathbb{G})$-Galois object. By Lemma 3.6 there exists $u \in \operatorname{Aut}_{g}(G)$ such that $(u, \bar{\sigma}) \in \Gamma(\mathbb{G})$, and hence by Proposition $3.3 A_{\sigma, a}^{u}(\mathbb{G})$ is $A(\mathbb{G})$-biGalois. By [15], Theorem 3.5, there 
exists $f \in \operatorname{Aut}_{\text {Hopf }}(A(\mathbb{G}))$ such that $Z={ }^{f} A_{\sigma, a}^{u}(\mathbb{G})$ as $A(\mathbb{G})$-bicomodule algebras, i.e. if $\beta^{\prime}$ denotes the left coaction of $Z$, we have $\beta^{\prime}=(f \otimes$ id $) \circ \beta_{u}$. There exists $v \in$ $\operatorname{Aut}_{g, \chi}(G)$ and $r \in \dot{k}$ such that $f(h)=v(h), \forall h \in G$ and $f(x)=r x$. Consider now a map $\mu: G \longrightarrow \dot{k}$ with $\mu(1)=1$ and $\mu(g)=r$. It is clear that $(v \circ u, \overline{\partial(\mu) \sigma}) \in \Gamma(\mathbb{G})$, and hence we consider the $A(\mathbb{G})$-biGalois extension $A_{\partial(\mu) \sigma, a}^{v \circ u}(\mathbb{G})$. It is not difficult to check that the right $A_{\sigma, a}(\mathbb{G})$-comodule algebra isomorphism $f^{\prime}: A_{\partial(\mu) \sigma, a}^{v \circ u}(\mathbb{G}) \longrightarrow$ ${ }^{f} A_{\sigma, a}^{u}(\mathbb{G})$ defined by $f^{\prime}(X)=X$ and $f^{\prime}\left(T_{h}\right)=\mu(h) T_{h}, \forall h \in G$, is also left colinear. Therefore $\Psi$ is also surjective

The type I case will be examined in more detail in the next section. The proof of the type II case is similar and left to the reader.

Proposition 3.9 Let $\mathbb{G}=(G, g, \chi)$ be a type II group datum. Then we have a group isomorphism:

$$
\begin{aligned}
\Psi: \Gamma(\mathbb{G}) & \longrightarrow \operatorname{BiGal}(A(\mathbb{G})) \\
(u, \bar{\sigma}) & \longmapsto\left[A_{\sigma, 0}^{u}\right] .
\end{aligned}
$$

We now begin the study of the biGalois group in the type IV case.

Proposition 3.10 Let $\mathbb{G}=(G, g, \chi)$ be a type $I V$ group datum. Let $\sigma \in Z^{2}(G, \dot{k})$. Consider the group morphism $\chi^{\prime}: G \longrightarrow \dot{k}$ defined by $\chi^{\prime}(h)=\sigma(g, h)^{-1} \sigma(h, g) \chi(h)$. Then the group datum $\mathbb{G}^{\prime}=\left(G, g, \chi^{\prime}\right)$ is a type IV group datum. The right $A(\mathbb{G})$ Galois object $A_{\sigma, 0}(\mathbb{G})$ is $A\left(\mathbb{G}^{\prime}\right)-A(\mathbb{G})$-biGalois. The Hopf algebras $A(\mathbb{G})$ and $A\left(\mathbb{G}^{\prime}\right)$ are isomorphic if and only if there exists $u \in \operatorname{Aut}_{g}(G)$ such that $(u, \bar{\sigma}) \in \Gamma(\mathbb{G})$.

Proof. It is easy to see that $\mathbb{G}^{\prime}$ is a type IV group datum and it is straightforward to check that there exists an algebra morphism $\beta: A_{\sigma, 0}(\mathbb{G}) \longrightarrow A\left(\mathbb{G}^{\prime}\right) \otimes A_{\sigma, 0}(\mathbb{G})$ such that $\beta(X)=1 \otimes X+x \otimes T_{g}$ and $\beta\left(T_{h}\right)=h \otimes T_{h}, \forall h \in G$, that endows $A_{\sigma, 0}(\mathbb{G})$ with an $A\left(\mathbb{G}^{\prime}\right)-A(\mathbb{G})$ bicomodule algebra structure. Very similarly to Proposition 3.3, we see that $A_{\sigma, 0}(\mathbb{G})$ is $A\left(\mathbb{G}^{\prime}\right)-A(\mathbb{G})$-biGalois. The Hopf algebras $A\left(\mathbb{G}^{\prime}\right)$ and $A(\mathbb{G})$ are isomorphic if and only if the corresponding group data are isomorphic, i.e. if and only if there exists $u \in \operatorname{Aut}_{g}(G)$ such that $\chi \circ u=\chi^{\prime}$. This last condition exactly means that $(u, \bar{\sigma}) \in \Gamma(\mathbb{G})$.

It is possible that the Hopf algebras $A(\mathbb{G})$ and $A\left(\mathbb{G}^{\prime}\right)$ are not isomorphic. Indeed we have the following example.

Example 3.11 Let $d>1$ and let $G=\left\langle x, h \mid x^{d^{4}}=1=h^{d^{2}}, x h=h x\right\rangle(G \cong$ $\left.C_{d^{4}} \times C_{d^{2}}\right)$. Let $g=x^{d}$ and let $q \in \dot{k}$ be a primitive $d^{2}$ th root of unity. Let $\chi: G \longrightarrow \dot{k}$ be the character defined by $\chi(x)=q$ and $\chi(h)=1$. We have $d=o(\chi(g))$, $d^{2}=o(\chi)$ and $d^{3}=o(g)$. For any $\sigma \in Z^{2}(G, \dot{k})$, we have $\sigma\left(g^{d}, x\right)=\sigma\left(x, g^{d}\right)$ while $\chi^{d}(x)=\chi(g) \neq 1$. Hence $\mathbb{G}=(G, g, \chi)$ is a type IV group datum.

Now consider $\sigma \in Z^{2}(G, \dot{k})$ defined by $\sigma\left(x^{\alpha} h^{\beta}, x^{\alpha^{\prime}} h^{\beta^{\prime}}\right)=q^{\alpha \beta^{\prime}}$ ( $\sigma$ is in fact a bicharacter). We have $\sigma(g, h)^{-1} \sigma(h, g)=q^{-1}$. Let $u: G \longrightarrow G$ be a group morphism. We have $u(h)=x^{\alpha} h^{\beta}$ and since $u\left(h^{d^{2}}\right)=1$, we have $d^{2} \mid \alpha$ and we conclude 
that $\chi \circ u(h)=1$. Hence there does not exist $u \in \operatorname{Aut}_{g}(G)$ such that $(u, \bar{\sigma}) \in \Gamma(\mathbb{G})$, and we conclude that the Hopf algebras $A(\mathbb{G})$ and $A\left(\mathbb{G}^{\prime}\right)$ are not isomorphic.

The following result concludes the proof of Theorem 3.2 in the type IV case. Since its proof is very similar to the one of Proposition 3.8, it is left to the reader.

Proposition 3.12 Let $\mathbb{G}=(G, g, \chi)$ be a type IV group datum. Then we have a group isomorphism:

$$
\begin{aligned}
\Psi: \Gamma(\mathbb{G}) & \longrightarrow \operatorname{BiGal}(A(\mathbb{G})) \\
(u, \bar{\sigma}) & \longmapsto\left[A_{\sigma, 0}^{u}\right] .
\end{aligned}
$$

We now study the case of type III group data. This is certainly the richest case.

Proposition 3.13 Let $\mathbb{G}=(G, g, \chi)$ be a type III group datum.

i) Let $\sigma \in Z^{2}(G, \dot{k})$. Consider the group morphism $\chi^{\prime}: G \longrightarrow \dot{k}$ defined by $\chi^{\prime}(h)=$ $\sigma(g, h)^{-1} \sigma(h, g) \chi(h)$ and the group datum $\mathbb{G}^{\prime}=\left(G, g, \chi^{\prime}\right)$. The group datum $\mathbb{G}^{\prime}$ is a type III or $V$ group datum. The right $A(\mathbb{G})$-Galois object $A_{\sigma, 0}(\mathbb{G})$ is $A\left(\mathbb{G}^{\prime}\right)-A(\mathbb{G})$ biGalois, and the Hopf algebras $A(\mathbb{G})$ and $A\left(\mathbb{G}^{\prime}\right)$ are isomorphic if and only if there exists $u \in \operatorname{Aut}_{g}(G)$ such that $(u, \bar{\sigma}) \in \Gamma(\mathbb{G})$.

ii) Let $\sigma \in Z^{2}(G, \dot{k})$ and let $a \in \dot{k}$ such that $A_{\sigma, a}(\mathbb{G})$ is $A(\mathbb{G})$-Galois. Consider the group morphism $\chi^{\prime}: G \longrightarrow \dot{k}$ defined by $\chi^{\prime}(h)=\sigma(g, h)^{-1} \sigma(h, g) \chi(h)$ and let $\mu=-a \sigma(g, g)^{-1} \ldots \sigma\left(g, g^{d-1}\right)^{-1}$. Then $\mathbb{G}^{\prime}=\left(G, g, \chi^{\prime}, \mu\right)$ is a type VI group datum, and $A_{\sigma, a}(\mathbb{G})$ is $A\left(\mathbb{G}^{\prime}\right)-A(\mathbb{G})$-biGalois.

Proof. i) The proof is similar to the one of Proposition 3.10.

ii) Since $A_{\sigma, a}(\mathbb{G})$ is $A(\mathbb{G})$-Galois, we have $\sigma\left(g^{d}, h\right)=\sigma\left(h, g^{d}\right), \forall h \in G$. Hence $\chi^{\prime d}=1$ and $\mathbb{G}^{\prime}$ is a type VI group datum. Then one checks that there exists an algebra morphism $\beta: A_{\sigma, a}(\mathbb{G}) \longrightarrow A\left(\mathbb{G}^{\prime}\right) \otimes A_{\sigma, a}(\mathbb{G})$ such that $\beta(X)=1 \otimes X+x \otimes T_{g}$ and $\beta\left(T_{h}\right)=h \otimes T_{h}, \forall h \in G$, that endows $A_{\sigma, a}(\mathbb{G})$ with an $A\left(\mathbb{G}^{\prime}\right)-A(\mathbb{G})$ bicomodule algebra structure. Very similarly to Proposition 3.3 , we see that $A_{\sigma, a}(\mathbb{G})$ is $A\left(\mathbb{G}^{\prime}\right)$ $A(\mathbb{G})$-biGalois.

The following two examples show that the group datum $\mathbb{G}^{\prime}$ of proposition 3.13.i might be of type III not isomorphic with $\mathbb{G}$, or of type V.

Example 3.14 Let $d>1$ and let $G=\left\langle g, h \mid g^{d^{2}}=1=h^{d}, g h=h g\right\rangle(G \cong$ $\left.C_{d^{2}} \times C_{d}\right)$. Let $q \in \dot{k}$ be a primitive $d$ th root of unity. Let $\chi: G \longrightarrow \dot{k}$ be the character defined by $\chi(g)=q$ and $\chi(h)=1$ : we have $g^{d} \neq 1$ and $\chi^{d}=1$ and hence $\mathbb{G}=(G, g, \chi)$ is a type III group datum.

Now consider $\sigma \in Z^{2}(G, \dot{k})$ defined by $\sigma\left(g^{\alpha} h^{\beta}, g^{\alpha^{\prime}} h^{\beta^{\prime}}\right)=q^{\alpha \beta^{\prime}}(\sigma$ is in fact a bicharacter). We have $\sigma(g, h)^{-1} \sigma(h, g)=q^{-1}$ and hence $\chi^{\prime d}=1$ : $\mathbb{G}^{\prime}=\left(G, g, \chi^{\prime}\right)$ is type III group datum. Let $u: G \longrightarrow G$ be a group morphism. We have $u(h)=g^{\alpha} h^{\beta}$ and since $u\left(h^{d}\right)=1$, we have $d \mid \alpha$ and we conclude that $\chi \circ u(h)=1 \neq q^{-1}$. Hence there does not exist $u \in \operatorname{Aut}_{g}(G)$ such that $(u, \bar{\sigma}) \in \Gamma(\mathbb{G})$, and we conclude that the Hopf algebras $A(\mathbb{G})$ and $A\left(\mathbb{G}^{\prime}\right)$ are not isomorphic. 
Example 3.15 Let $d>1$ and let $G=\left\langle g, h \mid g^{d^{2}}=1=h^{d^{2}}, g h=h g\right\rangle(G \cong$ $\left.C_{d^{2}} \times C_{d^{2}}\right)$. Let $q \in \dot{k}$ be a primitive $d^{2}$ th root of unity. Let $\chi: G \longrightarrow \dot{k}$ be the character defined by $\chi(g)=q^{d}$ and $\chi(h)=1$ : we have $g^{d} \neq 1$ and $\chi^{d}=1$ and hence $\mathbb{G}=(G, g, \chi)$ is a type III group datum.

Now consider $\sigma \in Z^{2}(G, \dot{k})$ defined by $\sigma\left(g^{\alpha} h^{\beta}, g^{\alpha^{\prime}} h^{\beta^{\prime}}\right)=q^{\alpha \beta^{\prime}}(\sigma$ is a bicharacter). We have $\sigma(g, h)^{-1} \sigma(h, g)=q^{-1}$ and hence $\chi^{\prime d}(h)=\sigma\left(g^{d}, h\right)^{-1} \sigma\left(h, g^{d}\right) \chi^{d}(h)=$ $\sigma\left(g^{d}, h\right)^{-1} \sigma\left(h, g^{d}\right)=q^{-d}$. Hence $\mathbb{G}^{\prime}=\left(G, g, \chi^{\prime}\right)$ is type $\mathrm{V}$ group datum.

Using Proposition 3.13, the proof of the description of the biGalois group in the type III case is the same has in the previous cases, and hence is left once again to the reader.

Proposition 3.16 Let $\mathbb{G}=(G, g, \chi)$ be a type III group datum. Then we have a group isomorphism:

$$
\begin{aligned}
\Psi: \Gamma(\mathbb{G}) & \longrightarrow \operatorname{BiGal}(A(\mathbb{G})) \\
(u, \bar{\sigma}) & \longmapsto\left[A_{\sigma, 0}^{u}\right] .
\end{aligned}
$$

Corollary 3.17 Let $\mathbb{G}=(G, g, \chi)$ be a type $V$ group datum, and let $\sigma \in Z^{2}(G, \dot{k})$ be such that $\chi^{d}(h)=\sigma\left(g^{d}, h\right) \sigma\left(h, g^{d}\right)^{-1}$. Consider the group morphism $\chi_{\sigma}: G \rightarrow \dot{k}$ defined by $\chi_{\sigma}(h)=\sigma(g, h)^{-1} \sigma(h, g) \chi(h)$. Then $\mathbb{G}_{\sigma}=\left(G, g, \chi_{\sigma}\right)$ is a type III group datum, there exists an $A(\mathbb{G})-A\left(\mathbb{G}_{\sigma}\right)$-biGalois extension and group isomorphisms

$$
\operatorname{BiGal}(A(\mathbb{G})) \cong \operatorname{BiGal}\left(A\left(\mathbb{G}_{\sigma}\right)\right) \cong \Gamma\left(\mathbb{G}_{\sigma}\right) \cong \Gamma(\mathbb{G}) .
$$

Proof. It is immediate that $\mathbb{G}_{\sigma}$ is a type III group datum. Consider now $\sigma^{-1} \in$ $Z^{2}(G, \dot{k})$. It is clear that the associated group datum $\left(\mathbb{G}_{\sigma}\right)^{\prime}$ of Proposition 3.13.i is $\mathbb{G}$, and hence that $A_{\sigma^{-1}, 0}(\mathbb{G})$ is $A(\mathbb{G})-A\left(\mathbb{G}_{\sigma}\right)$-biGalois, which gives the first isomorphism. The second isomorphism is given by Proposition 3.16. For the last one, it is not difficult to check that we have a map

$$
\begin{aligned}
\Gamma\left(\mathbb{G}_{\sigma}\right) & \longrightarrow \Gamma(\mathbb{G}) \\
(u, \bar{\tau}) & \longmapsto\left(u, \overline{(\sigma \circ u \times u)^{-1} \sigma \tau}\right)
\end{aligned}
$$

which is a group isomorphism.

This last corollary finally completes the proofs of Theorems 2.1 and 3.2.

Corollary 3.18 Let $\mathbb{G}=(G, g, \chi, \mu)$ be a type VI group datum. Consider the type III group datum $\mathbb{G}_{r e d}=(G, g, \chi)$. Then there exists an $A(\mathbb{G})-A\left(\mathbb{G}_{r e d}\right)$ biGalois extension, and hence a bijection

$$
\operatorname{Gal}(A(\mathbb{G})) \cong H^{2}(G, \dot{k}) \amalg H_{g^{d}, g^{d}}^{2}(G, \dot{k})
$$

and a group isomorphism

$$
\operatorname{BiGal}(A(\mathbb{G})) \cong \Gamma(\mathbb{G})
$$

Proof. It is clear from the second part of Proposition 3.13 that $A_{1,-\mu}\left(\mathbb{G}_{r e d}\right)$ is $A(\mathbb{G})-A\left(\mathbb{G}_{r e d}\right)$-biGalois. Hence the type III case of Theorems 2.1 and 3.2 conclude the proof. 


\section{Examples}

This section is devoted to the study of a few concrete examples. We first consider cyclic group data, and we get in particular Masuoka's description 12 of the Galois objects for the Taft algebras and Schauenburg's description [15] of their biGalois groups. Then we consider decomposable group data and get a more explicit description of corresponding biGalois groups: in particular we recover Schauenburg's description of the biGalois groups of the generalized Taft algebras, in a slightly different form.

\subsection{Cyclic group data}

A group datum $\mathbb{G}=(G, g, \chi, \mu)$ is said to be cyclic if the underlying group $G$ is cyclic. In this subsection we completely describe the Galois objects and biGalois groups for Hopf algebras associated with cyclic group data.

Before stating the main result, we need to introduce some terminology. Let $N>1$ be an integer and let $n_{1}, \ldots, n_{r}$ be some divisors of $N$. We put

$$
U(\mathbb{Z} / N \mathbb{Z})\left[n_{1}, \ldots, n_{r}\right]=\left\{\bar{\beta} \in U(\mathbb{Z} / N \mathbb{Z})(\beta \in \mathbb{Z}) \mid \beta \equiv 1\left(\bmod n_{i}\right), i=1, \ldots, r\right\} .
$$

This is clearly a subgroup of $U(\mathbb{Z} / N \mathbb{Z}) \cong \operatorname{Aut}\left(C_{N}\right)$. For $N=1$, we adopt the convention $U(\mathbb{Z} / N \mathbb{Z})=\{1\}$.

Theorem 4.1 Let $\mathbb{G}=(G, g, \chi, \mu)$ be a cyclic group datum with $d=o(\chi(g)), n=$ $o(g), N=|G|$ and $m=o(\chi)$.

- If $\mathbb{G}$ is a type I group datum, i.e. if $d=n=m$, then

$$
\operatorname{Gal}(A(\mathbb{G})) \cong \dot{k} / \dot{k}^{N} \times k \quad \text { and } \quad \operatorname{BiGal}(A(\mathbb{G})) \cong\left(\operatorname{Aut}\left(C_{\frac{N}{n}}\right) \ltimes\left(\dot{k} \times \dot{k} / \dot{k}^{\frac{N}{n}}\right)\right) \ltimes k .
$$

- If $\mathbb{G}$ is a type II group datum, i.e. if $d=n<m$, then

$$
\operatorname{Gal}(A(\mathbb{G})) \cong \dot{k} / \dot{k}^{N} \quad \text { and } \quad \operatorname{BiGal}(A(\mathbb{G})) \cong U(\mathbb{Z} / N \mathbb{Z})[m] \ltimes\left(\dot{k} \times \dot{k} / \dot{k}^{\frac{N}{n}}\right) .
$$

- If $\mathbb{G}$ is a type III or VI group datum, i.e. if $d=m<n$, then

$\operatorname{Gal}(A(\mathbb{G})) \cong \dot{k} / \dot{k}^{N} \amalg\left(\dot{k} \times \dot{k} / \dot{k}^{\frac{N d}{n}}\right) \quad$ and $\quad \operatorname{BiGal}(A(\mathbb{G})) \cong U(\mathbb{Z} / N \mathbb{Z})[n] \ltimes\left(\dot{k} \times \dot{k} / \dot{k}^{\frac{N}{n}}\right)$.

- If $\mathbb{G}$ is a type IV group datum, i.e. if $d<n$ and $d<m$, then

$$
\operatorname{Gal}(A(\mathbb{G})) \cong \dot{k} / \dot{k}^{N} \quad \text { and } \quad \operatorname{BiGal}(A(\mathbb{G})) \cong U(\mathbb{Z} / N \mathbb{Z})[n, m] \ltimes\left(\dot{k} \times \dot{k} / \dot{k}^{\frac{N}{n}}\right) .
$$

The rest of this subsection is essentially devoted to the proof of Theorem 4.1. The first remark is that since any 2-cocycle on a cyclic group is symmetric, there does not exist any type $\mathrm{V}$ cyclic group datum, and we have the following result.

Lemma 4.2 Let $\mathbb{G}=(G, g, \chi, \mu)$ be a cyclic group datum. Then

$$
\Gamma(\mathbb{G}) \cong \operatorname{Aut}_{g, \chi}(G) \ltimes H_{1, g}^{2}(G, \dot{k}) .
$$


The next step is to compute the modified cohomology groups of the first section in the case of a cyclic group. This is done in the following lemma.

Lemma 4.3 Let $C_{N}$ be the cyclic group of order $N$ and let $g \in C_{N}$ be an element of order $n>1$. Then we have a group isomorphism:

$$
H_{1, g}^{2}\left(C_{N}, \dot{k}\right)=H_{g, g}^{2}\left(C_{N}, \dot{k}\right) \cong \dot{k} \times\left(\dot{k} / \dot{k}^{\frac{N}{n}}\right) .
$$

Proof. We construct a split exact sequence

$$
1 \longrightarrow \dot{k} / \dot{k}^{\frac{N}{n}} \stackrel{u}{\longrightarrow} H_{1, g}^{2}(G, \dot{k}) \stackrel{\epsilon}{\longrightarrow} \dot{k} \longrightarrow 1
$$

where the group morphism $\epsilon: H_{1, g}^{2}(G, \dot{k}) \longrightarrow \dot{k}$ was constructed in subsection 1.3. Let us choose a generator $y$ of $C_{N}$ such that $g=y^{\frac{N}{n}}$. For $a \in \dot{k}$, define $f_{a} \in Z^{2}(G, \dot{k})$ in the following way [8]. For $0 \leq i, j \leq N-1$, we put $f_{a}\left(y^{i}, y^{j}\right)=1$ if $i+j \leq N-1$ and $f_{a}\left(y^{i}, y^{j}\right)=a$ if $i+j \geq N$. We have $\epsilon\left(\overline{f_{a}}\right)=a$ and hence the group morphism $f: \dot{k} \rightarrow H_{1, g}^{2}(G, \dot{k}), a \longmapsto \overline{f_{a}}$, satisfies $\epsilon \circ f=\mathrm{id}_{\dot{k}}$.

Let us fix a family of integers $\left(r_{i}\right)_{0 \leq i \leq N-1}$ with $r_{0}=1$ and $r_{\frac{N}{n}}=1$, and for $t \in \dot{k}$, let us define $\beta_{t}: G \longrightarrow \dot{k}$ by $\beta_{t}\left(y^{i}\right)=t^{r_{i}}$, for $0 \leq i \leq N-1$. We have $\beta_{t}(g)=t$. Now define a group morphism

$$
u_{0}: \dot{k} \longrightarrow H_{1, g}^{2}(G, \dot{k}), \quad t \longmapsto \overline{f_{t^{-n}} \partial\left(\beta_{t}\right)} .
$$

It is clear that $u_{0}(\dot{k}) \subset \operatorname{Ker}(\epsilon)$. Now let $\sigma \in Z^{2}(G, \dot{k})$ be such that $\bar{\sigma} \in \operatorname{Ker}(\epsilon)$. By the description of $H^{2}(G, \dot{k})$ in [8], Theorem 2.3.1, there exists $a \in \dot{k}$ and $\mu: G \rightarrow \dot{k}$ (with $\mu(1)=1)$ such that $\sigma=f_{a} \partial(\mu)$. Then $a=\mu(g)^{-n}$. Since $\beta_{\mu(g)}(g)=\mu(g)$, we have $\overline{\partial(\mu)}=\overline{\partial\left(\beta_{\mu(g)}\right)}$, and $\bar{\sigma}=\overline{f_{\mu(g)^{-n}} \partial\left(\beta_{\mu(g)}\right)} \in u(\dot{k})$. We conclude that $\operatorname{Ker}(\epsilon)=u_{0}(\dot{k})$.

Now let $t \in \dot{k}$ be such that $u_{0}(t)=\overline{1}$. Then there exists $\mu: G \rightarrow \dot{k}$ with $\mu(g)=1$ such that $f_{t^{-n}} \partial\left(\beta_{t}\right)=\partial(\mu)$, and hence $f_{t^{-n}}$ is a coboundary, which means that $t^{-n} \in \dot{k}^{N}\left([\underline{8})\right.$, i.e. that $t \in \dot{k}^{\frac{N}{n}}$ (recall that $k$ contains all primitive roots of unity). Conversely let $t \in \dot{k}^{\frac{N}{n}}$ and let $s \in \dot{k}$ be such that $t^{-n}=s^{N}$. Define $\gamma_{s}: G \longrightarrow \dot{k}$ by $\gamma_{s}\left(y^{i}\right)=s^{i}$ for $0 \leq i \leq N-1$. Then $f_{t^{-n}}=\partial\left(\gamma_{s}\right)$ and $f_{t^{-n}} \partial\left(\beta_{t}\right)=\partial\left(\gamma_{s} \beta_{t}\right)$, with $\gamma_{s} \beta_{t}(g)=s^{\frac{N}{n}} t=1$, and thus $t \in \operatorname{Ker}\left(u_{0}\right)$. Therefore $u_{0}$ induces an injective morphism $u: \dot{k} / \dot{k}^{\frac{N}{n}} \longrightarrow H_{1, g}^{2}(G, \dot{k})$ and we have the announced split exact sequence.

Let us now describe the explicit models for cyclic group data (we do not treat the type VI case thanks to Corollary 3.18). A cyclic datum is a 5-uplet $(d, n, N, \alpha, q)$ where $d, n, N>1$ are integers, $\alpha \in \mathbb{N}^{*}$ and $q \in \dot{k}$ is a root of unity, satisfying:

$$
d|n| N, \alpha \mid \frac{N}{n}, \operatorname{GCD}(\alpha, d)=1, o(q)=\frac{N d}{\alpha n} .
$$

To any cyclic datum $(d, n, N, \alpha, q)$ we associate a group datum

$$
\mathbb{C}[d, n, N, \alpha, q]:=\left(C_{N}=\langle z\rangle, g=z^{\frac{N}{n}}, \chi_{q}\right)
$$


where $\chi_{q}$ is the character defined by $\chi_{q}(z)=q$. The exact relations between cyclic data and cyclic group data are given by the following lemma.

Lemma 4.4 i) Let $(d, n, N, \alpha, q)$ be a cyclic datum. Then $\mathbb{C}[d, n, N, \alpha, q]$ is a cyclic group datum with $d=o\left(\chi_{q}(g)\right), n=o(g), N=|G|$ and $m=o\left(\chi_{q}\right)=\frac{N d}{\alpha n}$.

ii) Let $\mathbb{G}=(G, g, \chi)$ be a cyclic group datum. Then there exists a cyclic datum $(d, n, N, \alpha, q)$ such that $\mathbb{G} \cong \mathbb{C}[d, n, N, \alpha, q]$.

iii) Let $(d, n, N, \alpha, q)$ be a cyclic datum, with the cyclic group datum $\mathbb{C}[d, n, N, \alpha, q]$.

- $\mathbb{C}[d, n, N, \alpha, q]$ is a type I group datum if and only if

$$
d=N \quad \text { or } \quad\left[d=n<N, \operatorname{GCD}\left(\frac{N}{n}, n\right)=1 \text { and } \alpha=\frac{N}{n}\right] .
$$

- $\mathbb{C}[d, n, N, \alpha, q]$ is a type II group datum if and only if

$$
d=n<N \text { and } \alpha<\frac{N}{n} .
$$

- $\mathbb{C}[d, n, N, \alpha, q]$ is a type III group datum if and only if

$$
d<n=N \quad \text { or } \quad\left[d<n<N, \operatorname{GCD}\left(\frac{N}{n}, d\right)=1 \text { and } \alpha=\frac{N}{n}\right] .
$$

- $\mathbb{C}[d, n, N, \alpha, q]$ is a type IV group datum if and only if

$$
d<n<N \text { and } \alpha<\frac{N}{n} \text {. }
$$

Proof. i) We have

$$
o(\chi(g))=o\left(q^{\frac{N}{n}}\right)=\frac{o(q)}{\operatorname{GCD}\left(o(q), \frac{N}{n}\right)}=\frac{\frac{N d}{\alpha n}}{\operatorname{GCD}\left(\frac{N}{\alpha n} \alpha, \frac{N}{\alpha n} d\right)}=d
$$

since $\operatorname{GCD}(\alpha, d)=1$. The other assertions are immediate.

ii) Let $\mathbb{G}=(G, g, \chi)$ be a cyclic group datum with $|G|=N$ and $o(g)=n$ Let us choose a generator $z \in G$ such that $g=z^{\frac{N}{n}}$. Let $q=\chi(z)$. We have

$$
d=o(\chi(g))=o\left(q^{\frac{N}{n}}\right)=\frac{o(q)}{\operatorname{GCD}\left(o(q), \frac{N}{n}\right)} .
$$

From this we see that $o(q) \mid\left(\frac{N}{n} d\right)$. Let $\alpha \in \mathbb{N}^{*}$ be such that $\alpha o(q)=\frac{N}{n} d$. Then $\frac{o(q)}{d} \alpha=\frac{N}{n}$ and hence $\alpha \mid \frac{N}{n}$. Finally

$$
\frac{N}{n \alpha}=\operatorname{GCD}\left(o(q), \frac{N}{n}\right)=\operatorname{GCD}\left(\frac{N}{n \alpha} d, \frac{N}{n \alpha} \alpha\right)
$$

and we conclude that $\operatorname{GCD}(\alpha, d)=1$. Thus $(d, n, N, \alpha, q)$ is a cyclic datum and we have $\mathbb{G} \cong \mathbb{C}[d, n, N, \alpha, q]$. The final assertions are immediate. 
Proof of Theorem 4.1. Let $\mathbb{G}=(G, g, \chi)$ be a cyclic group datum. By Lemma 4.4 we can assume that

$$
\mathbb{G}=\mathbb{C}[d, n, N, \alpha, q]=\left(C_{N}=\langle z\rangle, g=z^{\frac{N}{n}}, \chi_{q}\right)
$$

for a cyclic datum $(d, n, N, \alpha, q)$. We begin with some generalities. We always have $H_{1, g}^{2}(G, \dot{k}) \cong \dot{k} \times \dot{k} / \dot{k}^{\frac{N}{n}}$ by Lemma 4.3. Let $u \in \operatorname{Aut}_{g, \chi}(G)$. Then there exists $\beta \in\{0, \ldots, N-1\}$ such that $u(z)=z^{\beta}$ with

$$
\operatorname{GCD}(\beta, N)=1, \quad n\left|(\beta-1), \quad \frac{N d}{\alpha n}\right|(\beta-1) .
$$

Hence we have

$$
\operatorname{Aut}_{g, \chi}(G) \cong U(\mathbb{Z} / N \mathbb{Z})\left[n, \frac{N d}{\alpha n}\right] \text { and } \Gamma(\mathbb{G}) \cong U(\mathbb{Z} / N \mathbb{Z})\left[n, \frac{N d}{\alpha n}\right] \ltimes\left(\dot{k} \times \dot{k} / \dot{k}^{\frac{N}{n}}\right)
$$

by Lemma 4.2. The proof of Theorem 4.1 now follows easily from Theorems 2.1, 3.2, Lemma 4.4 and the case by case computation of $U(\mathbb{Z} / N \mathbb{Z})\left[n, \frac{N d}{\alpha n}\right]$.

Let us specialize Theorem 4.1 to a few famous examples of Hopf algebras. First consider the cyclic datum $(N, N, N, 1, q)$. The cyclic group datum $\mathbb{C}[N, N, N, 1, q]$ is of type $\mathrm{I}$, and the corresponding Hopf algebra $A(\mathbb{C}[N, N, N, 1, q])$ is precisely the Taft algebra $H_{N, q}$. Hence we get Masuaoka's [12] and Schauenburg's [16] respective results

$$
\operatorname{Gal}\left(H_{N, q}\right) \cong \dot{k} / \dot{k}^{\frac{N}{n}} \text { and } \operatorname{BiGal}\left(H_{N, q}\right) \cong \dot{k} \ltimes k .
$$

Now consider the cyclic datum $(d, N, N, 1, q)$ with $d<N$. The cyclic group datum $\mathbb{C}[d, N, N, 1, q]$ is of type III, and hence for $\mu \in k$ we can consider the group datum $\mathbb{C}[d, N, N, 1, q][\mu]=\left(G=C_{N}=\langle g\rangle, g, \chi_{q}, \mu\right)$. The corresponding Hopf algebra $A(\mathbb{C}[d, N, N, 1, q][\mu])$ is, with the notation of [14], the simple-pointed Hopf algebra $A_{(q, \mu, d, N)}$ of [14]. Hence we get

$$
\operatorname{Gal}\left(A_{(q, \mu, d, N)}\right) \cong \dot{k} / \dot{k}^{N} \amalg\left(\dot{k} \times k / \dot{k}^{d}\right) \quad \text { and } \quad \operatorname{BiGal}\left(A_{(q, \mu, d, N)}\right) \cong \dot{k} .
$$

\subsection{Decomposable group data}

The first goal of this section is to provide a more concrete description for the biGalois group of the Hopf algebra associated to a type I group datum. We prove the following result.

Theorem 4.5 Let $\mathbb{G}=(G, g, \chi)$ be a type I group datum with $K=\operatorname{Ker}(\chi)$ and $d=o(\chi(g))=o(g)=o(\chi)$. Then we have a bijection

$$
\operatorname{Gal}(A(\mathbb{G})) \cong \dot{k} / \dot{k}^{d} \times H^{2}(K, \dot{k}) \times \operatorname{Hom}\left(K, \mu_{d}\right) \times k,
$$

and a group isomorphism

$$
\operatorname{BiGal}(A(\mathbb{G})) \cong \operatorname{Aut}(K) \ltimes\left((\dot{k} \ltimes k) \times H^{2}(K, \dot{k}) \times \operatorname{Hom}\left(K, \mu_{d}\right)\right)
$$

where the right actions of $\operatorname{Aut}(K)$ on $H^{2}(K, \dot{k})$ and $\operatorname{Hom}\left(K, \mu_{d}\right)$ are the natural ones, and the action on $\dot{k} \ltimes k$ is the trivial one. 
In fact the comparison of our description of the biGalois group of a generalized Taft algebra with Schauenburg's one [16] will lead us to consider more generally decomposable group data. We say that a group datum $\mathbb{G}=(G, g, \chi, \mu)$ is decomposable if $G$ is the direct product of $\langle g\rangle$ with a subgroup $K$. It is easy to see that $\mathbb{G}$ is a type I group datum if and only if $\mu=0$ and $\mathbb{G}$ is decomposable with $G$ being the direct product of $\langle g\rangle$ and of $K=\operatorname{ker}(\chi)$.

The first step when studying decomposable group data is to examine the behaviour of the modified cohomology group under direct product. We have the following slight generalization of a theorem of Yamazaki, see [8], Theorem 2.3.13. For groups $G_{1}$ and $G_{2}$, the set of pairings (i.e. bimorphisms) $G_{1} \times G_{2} \longrightarrow \dot{k}$ is denoted by $\mathcal{P}\left(G_{1} \times G_{2}, \dot{k}\right)$. It has a natural group structure.

Lemma 4.6 Let $G_{1}, G_{2}$ be some groups and let $g, h \in Z\left(G_{1}\right)$. Then we have a group isomorphism

$$
H_{g, h}^{2}\left(G_{1} \times G_{2}, \dot{k}\right) \cong H_{g, h}^{2}\left(G_{1}, \dot{k}\right) \times H^{2}\left(G_{2}, \dot{k}\right) \times \mathcal{P}\left(G_{1} /\langle g\rangle \times G_{2}, \dot{k}\right) .
$$

Proof. We freely use the techniques and results of the proof of Theorem 2.3.13 in [8]. Let us first consider the group morphism

$$
\begin{aligned}
\Psi_{0}: Z_{g}^{2}\left(G_{1} \times G_{2}, \dot{k}\right) & \longrightarrow Z_{g}^{2}\left(G_{1}, \dot{k}\right) \times Z^{2}\left(G_{2}, \dot{k}\right) \times \mathcal{P}\left(G_{1} /\langle g\rangle \times G_{2}, \dot{k}\right) \\
\sigma & \longmapsto\left(\sigma_{1}, \sigma_{2}, B_{\sigma}\right)
\end{aligned}
$$

where $\sigma_{i}, i=1,2$, is the restriction of $\sigma$ to $G_{i}$ and $B_{\sigma}$ is the pairing defined by $B_{\sigma}\left(\overline{g_{1}}, g_{2}\right)=\sigma\left(g_{1}, g_{2}\right) \sigma\left(g_{2}, g_{1}\right)^{-1}, \forall\left(g_{1}, g_{2}\right) \in G_{1} \times G_{2}$. Clearly $\Psi_{0}$ induces a group morphism

$$
\begin{aligned}
\Psi: H_{g, h}^{2}\left(G_{1} \times G_{2}, \dot{k}\right) & \longrightarrow H_{g, h}^{2}\left(G_{1}, \dot{k}\right) \times H^{2}\left(G_{2}, \dot{k}\right) \times \mathcal{P}\left(G_{1} /\langle g\rangle \times G_{2}, \dot{k}\right) \\
\bar{\sigma} & \longmapsto\left(\overline{\sigma_{1}}, \overline{\sigma_{2}}, B_{\sigma}\right) .
\end{aligned}
$$

For $\left(\sigma_{1}, \sigma_{2}, B\right) \in Z_{g}^{2}\left(G_{1}, \dot{k}\right) \times Z^{2}\left(G_{2}, \dot{k}\right) \times \mathcal{P}\left(G_{1} /\langle g\rangle \times G_{2}, \dot{k}\right)$, define an element $\sigma \in Z_{g}^{2}\left(G_{1} \times G_{2}, \dot{k}\right)$ by

$$
\sigma\left(g_{1} g_{2}, g_{1}^{\prime} g_{2}^{\prime}\right)=\sigma_{1}\left(g_{1}, g_{1}^{\prime}\right) \sigma_{2}\left(g_{2}, g_{2}^{\prime}\right) B\left(\overline{g_{1}}, g_{2}^{\prime}\right), \forall g_{1}, g_{1}^{\prime} \in G_{1}, g_{2}, g_{2}^{\prime} \in G_{2} .
$$

Clearly $\Psi(\bar{\sigma})=\left(\overline{\sigma_{1}}, \overline{\sigma_{2}}, B_{\sigma}\right)$, and hence $\Psi$ is surjective. Now let $\sigma \in Z_{g}^{2}\left(G_{1} \times G_{2}, \dot{k}\right)$ be such that $\bar{\sigma} \in \operatorname{Ker}(\Psi)$. This means that there exists $\mu_{1}: G_{1} \rightarrow \dot{k}$ with $\mu_{1}(h)=$ $1=\mu_{1}(1), \mu_{2}: G_{2} \rightarrow \dot{k}$ with $\mu_{2}(1)=1$, and that $B_{\sigma}=1$. Now define a map

$$
\mu: G_{1} \times G_{2} \rightarrow \dot{k} \text { by } \mu\left(g_{1} g_{2}\right)=\mu_{1}\left(g_{1}\right) \mu_{2}\left(g_{2}\right), \forall\left(g_{1}, g_{2}\right) \in G_{1} \times G_{2} .
$$

We have $\mu(h)=1$ and hence $\bar{\sigma}=\overline{\partial(\mu) \sigma}$. In this way we can assume without loss of generality that $\sigma\left(g_{1}, g_{1}^{\prime}\right)=1=\sigma\left(g_{2}, g_{2}^{\prime}\right), \forall g_{1}, g_{1}^{\prime} \in G_{1}, g_{2}, g_{2}^{\prime} \in G_{2}$. Now consider the map $s: G_{1} \times G_{2} \rightarrow \dot{k}$ defined by $s\left(g_{1}, g_{2}\right)=\sigma\left(g_{1}, g_{2}\right)^{-1}$. By the computation in the proof of Theorem 2.3.13 in [8] (p. 61), we have $\sigma=\partial(s)$, and since $s(h)=1$, we conclude that $\bar{\sigma}=\overline{1}$ and that $\Psi$ is injective. 
Using this lemma, or simply Yamazaki's result in the type I, II and IV cases, one gets a more precise description for the set $\operatorname{Gal}(A(\mathbb{G}))$ of a decomposable group datum. The type I case is contained in the statement of Theorem 4.5, and we have:

Corollary 4.7 Let $\mathbb{G}=(G, g, \chi)$ be a decomposable group datum with $G=\langle g\rangle \times K$, and $d=o(\chi(g)), n=o(g)$. Assume that $\mathbb{G}$ is not a type I group datum.

- If $\mathbb{G}$ is of type II or IV, then

$$
\operatorname{Gal}(A(\mathbb{G})) \cong \dot{k} / \dot{k}^{n} \times H^{2}(K, \dot{k}) \times \operatorname{Hom}\left(K, \mu_{n}\right)
$$

- If $\mathbb{G}$ is of type III, $V$ or VI, then

$\operatorname{Gal}(A(\mathbb{G})) \cong\left(\dot{k} / \dot{k}^{n} \times H^{2}(K, \dot{k}) \times \operatorname{Hom}\left(K, \mu_{n}\right)\right) \amalg\left(\dot{k} \times \dot{k} / \dot{k}^{d} \times H^{2}(K, \dot{k}) \times \operatorname{Hom}\left(K, \mu_{n}\right)\right)$.

We now study the biGalois group of a type I group datum.

Lemma 4.8 Let $\mathbb{G}=(G, g, \chi)$ be a type I group datum, let $K=\operatorname{Ker}(\chi)$ and let $p_{2}: G \longrightarrow K$ be the canonical projection. The map

$$
\begin{aligned}
\Omega: \Gamma(\mathbb{G}) & \longrightarrow \operatorname{Aut}(K) \ltimes\left(\dot{k} \times H^{2}(K, \dot{k}) \times \operatorname{Hom}\left(K, \mu_{d}\right)\right) \\
(u, \bar{\sigma}) & \longmapsto\left(p_{2} \circ u_{\mid K}, \sigma(g, g) \ldots \sigma\left(g, g^{d-1}\right), \overline{\sigma_{\mid K \times K}}, \chi \circ u_{\mid K}\right)
\end{aligned}
$$

is a group isomorphism.

Proof. It is straightforward to check that $\Omega$ is well defined (is fact on the whole group $\left.\operatorname{Aut}_{g}(G) \ltimes H_{1, g}^{2}(G, \dot{k})\right)$ and is a group morphism. Let $(u, \bar{\sigma}) \in \mathrm{Ker} \Omega$. It is easy to see that, since $G=\langle g\rangle K$ and $K=\operatorname{Ker}(\chi)$, that $u=\operatorname{id}_{G}$. Since $(u, \bar{\sigma}) \in \Gamma(\mathbb{G})$, we have $\chi \circ u(h)=\sigma(g, h)^{-1} \sigma(h, g) \chi(h), \forall h \in G$, and hence $\sigma(g, h)=\sigma(h, g), \forall h \in G$. Thus the pairing $B_{\sigma}$ in the proof of Lemma 4.6 is trivial. By Lemma 4.3 the identity $\sigma(g, g) \ldots \sigma\left(g, g^{d-1}\right)=1$ precisely means that the restriction of $\sigma$ to $\langle g\rangle \times\langle g\rangle$ is trivial in $H_{1, g}^{2}(G, \dot{k})$, and we conclude that $\bar{\sigma}=\overline{1}$ by Lemma $4.6: \Omega$ is injective.

Finally let $(f, \lambda, \bar{\tau}, \psi) \in \operatorname{Aut}(K) \times \dot{k} \times H^{2}(K, \dot{k}) \times \operatorname{Hom}\left(K, \mu_{d}\right)$. Let $s: \mu_{d} \rightarrow\langle g\rangle$ be a group morphism such that $\chi \circ s=\mathrm{id}$. Define $u \in \operatorname{Aut}_{g}(G)$ by $u(g)=g$ and $u(h)=s(\psi(h)) f(h)$ for $h \in K$. Let $f_{\lambda} \in Z^{2}(\langle g\rangle, \dot{k})$ defined in the proof of Lemma 4.3, and define $\sigma \in Z^{2}(G, \dot{k})$ as in the proof of Lemma 4.6:

$$
\sigma\left(g^{\alpha} h, g^{\beta} h^{\prime}\right)=f_{\lambda}\left(g^{\alpha}, g^{\beta}\right) \tau\left(h, h^{\prime}\right) \psi\left(h^{\prime}\right)^{\alpha}, \forall \alpha, \beta \in \mathbb{Z}, \forall h, h^{\prime} \in K .
$$

It is clear that $(u, \bar{\sigma}) \in \Gamma(\mathbb{G})$ and that $\Omega(u, \bar{\sigma})=(f, \lambda, \bar{\tau}, \psi)$. Thus $\Omega$ is an isomorphism.

Theorem 4.5 is now a straightforward consequence of Theorem 3.2 and Lemma 4.8 .

Before coming to the generalized Taft algebras, let us examine the case of a type I group datum $\mathbb{G}=(G, g, \chi)$ where $K=\operatorname{Ker}(\chi)$ is an abelian group, written as a direct product of cyclic groups $K=\prod_{i=1}^{m} C_{N_{i}}$. We have a group isomorphism

$$
H^{2}(K, \dot{k}) \cong \prod_{i=1}^{m} H^{2}\left(C_{N_{i}}, \dot{k}\right) \times \operatorname{Hom}\left(\Lambda^{2}(K), \dot{k}\right),
$$


where $\operatorname{Hom}\left(\Lambda^{2}(K), \dot{k}\right)$ is the group of alternating bimorphism $B: K \times K \rightarrow \dot{k}$ (i.e. with $B(r, r)=1$ for $r \in K)$. This isomorphism is $\operatorname{Aut}(K)$-equivariant, the action of $\operatorname{Aut}(K)$ on $\operatorname{Hom}\left(\Lambda^{2}(K), \dot{k}\right)$ being the natural one while we need a few words to explain the action on $\prod_{i=1}^{m} H^{2}\left(C_{N_{i}}, \dot{k}\right)$. Let $\underline{\sigma}=\left(\overline{\sigma_{i}}\right) \in \prod_{i=1}^{m} H^{2}\left(C_{N_{i}}, \dot{k}\right)$ and let $u \in \operatorname{Aut}(K)$ written in a matrix form $u=\left(u_{i j}\right)$ with $u_{i j} \in \operatorname{Hom}\left(G_{j}, G_{i}\right)$. Then

$$
\underline{\sigma} \leftarrow u=\left(\prod_{j} \overline{\sigma_{j} \circ\left(u_{j i} \times u_{j i}\right)}\right)_{i}
$$

Thus by Theorem 4.5 we get a bijection

$$
\operatorname{Gal}(A(\mathbb{G})) \cong \dot{k} / \dot{k}^{d} \times\left(\prod_{i=1}^{m} \dot{k} / \dot{k}^{N_{i}}\right) \times \operatorname{Hom}\left(\Lambda^{2}(K), \dot{k}\right) \times \operatorname{Hom}\left(K, \mu_{d}\right) \times k,
$$

and a group isomorphism

$\operatorname{BiGal}(A(\mathbb{G})) \cong \operatorname{Aut}(K) \ltimes\left((\dot{k} \ltimes k) \times\left(\prod_{i=1}^{m} \dot{k} / \dot{k}^{N_{i}}\right) \times \operatorname{Hom}\left(\Lambda^{2}(K), \dot{k}\right) \times \operatorname{Hom}\left(K, \mu_{d}\right)\right)$.

The generalized Taft algebra $H_{N, m+1, q}$ with $(m+1)$ grouplike $(m \in \mathbb{N})$ may be defined as the Hopf algebra associated with the group datum $\mathbb{G}=\left(C_{N}^{m+1}, g, \chi_{q}\right)$ where $g$ is a generator of the first cyclic copy of $C_{N}^{m+1}, q$ is a primitive $N$ th root of unity and $\chi_{q}$ is the character defined by $\chi_{q}(g)=q$ and $\operatorname{Ker}(\chi)=C_{N}^{m}$. We get, after standard identifications, the result of Schauenburg [16]

$$
\operatorname{Gal}\left(H_{N, m+1, q}\right) \cong \dot{k} / \dot{k}^{N} \times\left(\dot{k} / \dot{k}^{N}\right)^{m} \times \operatorname{Skew}_{m}(\mathbb{Z} / N \mathbb{Z}) \times(\mathbb{Z} / N \mathbb{Z})^{m} \times k,
$$

(where $\operatorname{Skew}_{m}(\mathbb{Z} / N \mathbb{Z})$ is the set of $\mathbb{Z} / N \mathbb{Z}$-valued skew-symmetric matrices $r=\left(r_{i j}\right)$, i.e. with $r_{i j}=-r_{j i}$ and $\left.r_{i i}=0\right)$ and a group isomorphism

$\operatorname{BiGal}\left(H_{N, m+1, q}\right) \cong \mathrm{GL}_{m}(\mathbb{Z} / N \mathbb{Z}) \ltimes\left((\dot{k} \ltimes k) \times\left(\dot{k} / \dot{k}^{N}\right)^{m} \times \operatorname{Skew}_{m}(\mathbb{Z} / N \mathbb{Z}) \times(\mathbb{Z} / N \mathbb{Z})^{m}\right)$,

The right actions of $\mathrm{GL}_{m}(\mathbb{Z} / N \mathbb{Z})$ on each copy of the right side being the following ones. The action on $\dot{k} \ltimes k$ is the trivial one, the action on $\left(\dot{k} / \dot{k}^{N}\right)$ is the one induced by the $\mathbb{Z} / N \mathbb{Z}$-module structure of $\dot{k} / \dot{k}^{N}$ (see [16]), and the (right) actions on $\operatorname{Skew}_{m}(\mathbb{Z} / N \mathbb{Z})$ and $(\mathbb{Z} / N \mathbb{Z})^{m}$ are the natural ones (see also [16]).

Our description of the group structure of the biGalois group is different and seems to be simpler than the one of Schauenburg in [16], who used both an action (of $\left.\mathrm{GL}_{m}(\mathbb{Z} / N \mathbb{Z}) \ltimes(\mathbb{Z} / N \mathbb{Z})^{m}\right)$ and a cocycle to describe the group structure. Let us try to understand the reasons leading to the use of a cocycle.

So let us consider a decomposable group datum $\mathbb{G}=(G, g, \chi)$ where $G$ is the direct product $G=\langle g\rangle \times K$. We assume that $\chi^{n}=1$ where $n=o(g)$. An element of $\Gamma(\mathbb{G})$ may be described as an element

$$
\begin{gathered}
\left(f, \psi_{1}, \lambda, \bar{\sigma}, \psi_{2}\right) \in \operatorname{Aut}(K) \times \operatorname{Hom}(K,\langle g\rangle) \times \dot{k} \times H^{2}(K, \dot{k}) \times \operatorname{Hom}\left(K, \mu_{n}\right) \\
\text { satisfying } \chi(f(h)) \chi\left(\psi_{1}(h)\right)=\chi(h) \psi_{2}(h), \forall h \in K .
\end{gathered}
$$


Hence we see that since $\chi^{n}=1$, the element $\psi_{2}$ is redundant, and as a set we have

$$
\Gamma(\mathbb{G}) \cong \operatorname{Aut}(K) \times \operatorname{Hom}(K,\langle g\rangle) \times \dot{k} \times H^{2}(K, \dot{k}) .
$$

The group law is not the one of a simple semi-direct product, and this is where cocycles come into play for the description of the group structure. However when $K=\operatorname{Ker}(\chi)$, then $\psi_{1}$ is redundant with respect to the other data, and in this particular setting the group law is the one of a semi-direct product, as described in Theorem 4.5.

\section{References}

[1] N. AndruskiewitsCh, H.-J. Schneider, Lifting of quantum linear spaces and pointed Hopf algebras of dimension $p^{3}$, J. Algebra 209 (1998), 658-691.

[2] G.M. Bergman, The diamond lemma for ring theory, Adv. Math. 29 (1978), 178-218.

[3] J. Bichon, The representation category of the quantum group of a non-degenerate bilinear form, Comm. Algebra 31, No. 10 (2003), 4831-4851.

[4] C. Calinescu, S. Dascalescu, A. Masuoka, C. Meneni, Quantum lines over noncommutative cosemisimple Hopf algebras, J. Algebra 273 (2004), 753-779.

[5] P. Etingof, S. Gelaki, On cotriangular Hopf algebras, Amer. J. Math 123, No. 4 (2001), 699-713.

[6] X.-W. Chen, H.-L. Huang, Y. Ye, P. Zhang, Monomial Hopf algebras, Preprint arXiv:math.QA/0308023v2 (2003).

[7] C. Cibils, M. Rosso, Hopf quivers, J. Algebra 254 (2002), 241-251.

[8] G. Karpilovsky, Projective representations of finite groups, Marcel Dekker, 1985.

[9] R. GÜnther, Crossed products for pointed Hopf algebras, Comm. Algebra 27, No. 9 (1999), 4389-4410.

[10] C. Kassel, H.-J. Schneider, Homotopy theory of Hopf-Galois extensions, Preprint arXiv:math.QA/0402034 (2004).

[11] H.F. Kreimer, P.M. Cook II, Galois theories and normal bases, J. Algebra 43 (1976), $115-121$.

[12] A. Masuoka, Cleft extensions for a Hopf algebra generated by a nearly primitive element, Comm. Algebra 22, No. 11 (1994), 4537-4559.

[13] S. Montgomery, Hopf algebras and their actions on rings, Amer. Math. Soc., Providence, 1993.

[14] D.E. RADFord, Finite-dimensional simple-pointed Hopf algebras, J. Algebra 211 (1999), 686-710.

[15] P. Schauenburg, Hopf bigalois extensions, Comm. Algebra 24, No. 12 (1996), 3797 3825 . 
[16] P. Schauenburg, BiGalois objects over the Taft algebras, Israel J. Math. 115 (2000), 101-123.

[17] E.J. TAFT, The order of the antipode of finite-dimensional Hopf algebras, Proc. Nat. Acad of Sci. USA 68, No. 11 (1971), 2631-2633.

[18] K.H. UlbRICH, Fibre functors on finite dimensional comodules, Manuscripta Math. 65 (1989), 39-46. 